\title{
THE HEAT FLOW ON METRIC RANDOM WALK SPACES
}

\author{
JOSÉ M. MAZÓN, MARCOS SOLERA AND JULIÁN TOLEDO
}

\begin{abstract}
In this paper we study the Heat Flow on Metric Random Walk Spaces, which unifies into a broad framework the heat flow on locally finite weighted connected graphs, the heat flow determined by finite Markov chains and some nonlocal evolution problems. We give different characterizations of the ergodicity and prove that a metric random walk space with positive Ollivier-Ricci curvature is ergodic. Furthermore, we prove a Cheeger inequality and, as a consequence, we show that a Poincaré inequality holds if, and only if, an isoperimetric inequality holds. We also study the Bakry-Émery curvaturedimension condition and its relation with functional inequalities like the Poincaré inequality and the transport-information inequalities.
\end{abstract}

\section{Contents}

1. Introduction and Preliminaries

1.1. Metric Random Walk Spaces

1.2. Ollivier-Ricci Curvature

2. The Heat Flow on Metric Random Walk Spaces

2.1. The Heat Flow

2.2. Infinite Speed of Propagation and Ergodicity

3. Functional Inequalities

3.1. Spectral Gap and Poincaré Inequality

3.2. Isoperimetric Inequality

3.3. Cheeger Inequality

3.4. Spectral Gap and Curvature

3.5. Transport Inequalities

References

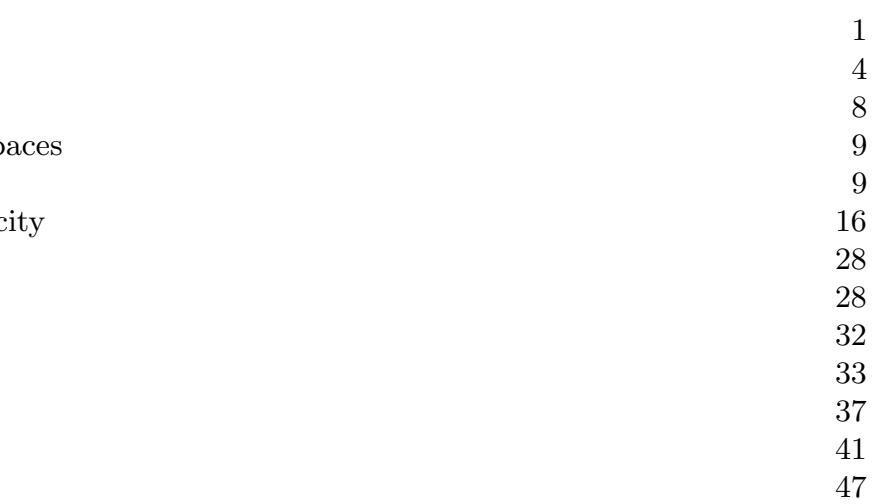

\section{Introduction and Preliminaries}

A metric random walk space is a metric space $(X, d)$ together with a family $m=\left(m_{x}\right)_{x \in X}$ of probability measures that encode the jumps of a Markov chain. Given an initial mass distribution $\mu$ on $X$, the measure $\mu * m$ given by

$$
\mu * m(A):=\int_{X} m_{x}(A) d \mu(x), \quad \text { for all Borel sets } A \subset X,
$$

Date: December 17, 2019.

Key words and phrases. Random walk, nonlocal operators, Logarithmic-Sobolev inequalities, Cheeger inequality, Ollivier-Ricci curvature, Bakry-Émery curvature-dimension condition, Concentration of measures, Transport inequalities 2010 Mathematics Subject Classification: 35K05, 47D07, 05C81, 31C20, 26D10, 45C99. 
describes the new mass distribution after a jump. Associated with $m$, the Laplace operator $\Delta_{m}$ is defined as

$$
\Delta_{m} f(x):=\int_{X}(f(y)-f(x)) d m_{x}(y) .
$$

Assuming that there exists an invariant and reversible measure $\nu$ for the random walk, the operator $-\Delta_{m}$ generates in $L^{2}(X, \nu)$ a Markovian semigroup $\left(e^{t \Delta_{m}}\right)_{t \geq 0}$ (Theorem 2.1) called the heat flow on the metric random walk space, which unifies into a broad framework the heat flow on graphs, the heat flow determined by finite Markov chains and also some nonlocal heat flows.

It is of great importance in many applications to understand the behaviour of the semigroup $\left(e^{t \Delta_{m}}\right)_{t \geq 0}$ as $t \rightarrow \infty$. In this regard, we introduce a new concept, called random walk connectedness or $m$ connectedness of the metric random walk space, which is related to the geometry of the metric random walk space. We then prove that it is equivalent to the infinite speed of propagation of the heat flow (Theorem 2.9) and also to the ergodicity of the Laplacian (Theorem 2.19), that in this context means that the only solutions of the equation $\Delta_{m} f=0$ are the constant functions, recall further that this is, in turn, equivalent to the ergodicity of the measure $\nu$ (see also Theorem 2.21). Moreover, we relate it with geometric properties of the metric random walk space (Theorem 2.24).

In 1969 Jeff Cheeger [17] proved his famous inequality

$$
\frac{h_{M}^{2}}{2} \leq \lambda_{1}\left(\Delta_{M}\right)
$$

where $\lambda_{1}\left(\Delta_{M}\right)$ is the first non-trivial eigenvalue of the Laplace Beltrami operator $\Delta_{M}$ on $L^{2}(M$, vol) of a compact manifold $M$ and the Cheeger constant $h_{M}$ is defined as

$$
h_{M}=\inf \frac{\operatorname{Area}(\partial S)}{\min (\operatorname{vol}(S), \operatorname{vol}(M \backslash S))},
$$

where the infimum runs over all $S \subset M$ with sufficiently smooth boundary. This inequality can be traced back to the paper by Polya and Szego [47]. The first Cheeger estimates on graphs are due to Dodziuk [21] and Alon and Milmann [2]. Since then, these estimates have been improved and various variants have been proved. For locally finite weighted connected graphs, the following relation between the Cheeger constant and the first positive eigenvalue $\lambda_{1}(G)$ of the graph Laplacian has been proved in [19] (see also [10])

$$
\frac{h_{G}^{2}}{2} \leq \lambda_{1}(G) \leq 2 h_{G},
$$

where $h_{G}$ is the Cheeger constant for graphs. For a general metric random walk space $[X, d, m]$ we define the Cheeger constant $h_{m}(X)$ and we obtain the Cheeger inequality (Theorem 3.12)

$$
\frac{h_{m}^{2}}{2} \leq \operatorname{gap}\left(-\Delta_{m}\right) \leq 2 h_{m},
$$

where $\operatorname{gap}\left(-\Delta_{m}\right)$ is the spectral gap of the Laplace operator. As a consequence, we show that a Poincaré inequality holds if, and only if, an isoperimetric inequality holds.

An important tool in the study of the speed of convergence of the heat flow to the equilibrium is the Poincaré inequality (see [6]). In the case of Riemannian manifolds and Markov diffusion semigroups, a usual condition required to obtain this functional inequality is the positivity of the corresponding Ricci curvature of the underlying space (see [6], [55]). In [7], Bakry and Emery found a way to define the lower Ricci curvature bound through the heat flow. Moreover, Renesse and Sturm [49] proved that, on a Riemannian manifold $M$, the Ricci curvature is bounded from below by some constant $K \in \mathbb{R}$ if, 
and only if, the Boltzmann-Shannon entropy is $K$-convex along geodesics in the 2-Wasserstein space of probability measures on $M$. This was the key observation, used simultaneously by Lott and Villani [34] and Sturm [51], to give a notion of a lower Ricci curvature bound in the general context of length metric measure spaces. In these spaces, the relation between the Bakry-Émery curvature-dimension condition and the notion of the Ricci curvature bound introduced by Lott-Villani-Sturm, was done by Ambrosio, Gigli and Savaré in [3], where they proved that these two notions of Ricci curvature coincide under certain assumptions on the metric measure space.

When the space under consideration is discrete, for instance, in the case of a graph, the previous concept of a Ricci curvature bound is not as clearly applicable as in the continuous setting. Indeed, the definition by Lott-Sturm-Villani does not apply if the 2-Wasserstein space over the metric measure space does not contain geodesics. Unfortunately, this is the case if the underlying space is discrete. Recently, Erbas and Maas [23], in the framework of Markov chains on discrete spaces, in order to circumvent the nonexistence of 2-Wasserstein geodesics, replace the 2-Wasserstein metric by a different metric, which was introduced by Maas in [37]. Here, we do not consider this notion of Ricci curvature bound which, in the framework of metric random walk spaces, will be the object of the forthcoming paper [40]. Instead, we will use two other concepts of a Ricci curvature bound, the one based on the Bakry-Émery curvaturedimension condition and the one introduced by Y. Ollivier in [43]. We refer to [42] and the references therein for the vibrant research field of discrete curvature.

The use of the Bakry-Émery curvature-dimension condition to obtain a possible definition of a Ricci curvature bound in Markov chains was first considered in 1998 by Schmuckenschlager [50]. Moreover, in 2010, Lin and Yau [33] used this concept for graphs. Subsequently, this concept of curvature in the discrete setting has been frequently used (see [31] and the references therein). Note that, to deal with the Bakry-Émery curvature-dimension condition, one needs a Carré du champ $\Gamma$. In the framework of Markov diffusion semigroups in order to get good inequalities from this curvature-dimension condition it is essential that the generator $A$ of the semigroup satisfies the chain rule formula

$$
A(\Phi(f))=\Phi^{\prime}(f) A(f)+\Phi^{\prime \prime}(f) \Gamma(f),
$$

which characterizes diffusion operators in the continuous setting (see [6]). Unfortunately, this chain rule does not hold in the discrete setting and this is one of the main difficulties when working with this curvature-dimension condition in metric random walk spaces.

In Riemannian geometry, positive Ricci curvature is characterized by the fact that "small balls are closer, in the 1-Wasserstein distance, than their centers are" (see [49]). In the framework of metric random walk spaces, inspired by this, Y. Ollivier [43] introduced the concept of coarse Ricci curvature, substituting the balls by the measures $m_{x}$. Moreover, he proved that positive coarse Ricci curvature implies positivity of the spectral gap. In Section 3 we give conditions on the Laplace operator $\Delta_{m}$ which ensure the positivity of the spectral gap and we relate bounds on the spectral gap with bounds on the Bakry-Émery curvature-dimension condition.

Following the papers by Marton and Talagrand ([36], [53]) about transport inequalities that relate Wasserstein distances with entropy and information, this research topic has had a great development (see the survey [27]). One of the keystones of this theory was the discovery in 1986 by Marton [35] of the link between transport inequalities and the concentration of measure. Concentration of measure inequalities can be obtained by means of other functional inequalities such as isoperimetric and logarithmic Sobolev inequalities, see the textbook by Ledoux [32] for an excellent account on the subject. We show that under the positivity of the Bakry-Émery curvature-dimension condition or the Ollivier-Ricci curvature a 
transport-information inequality holds (Theorems 3.22 and 3.28). Moreover, we prove that if a transportinformation inequality holds then a transport-entropy inequality is also satisfied (Theorem 3.25) and that, in general, the converse implication does not hold.

1.1. Metric Random Walk Spaces. Let $(X, d)$ be a Polish metric space equipped with its Borel $\sigma$-algebra.

Definition 1.1. A random walk $m$ on $X$ is a family of probability measures $m_{x}$ on $X, x \in X$, satisfying the following two technical conditions:

(i) the measures $m_{x}$ depend measurably on the point $x \in X$, i.e., for any Borel subset $A$ of $X$ and any Borel subset $B$ of $\mathbb{R}$, the set $\left\{x \in X: m_{x}(A) \in B\right\}$ is Borel,

(ii) each measure $m_{x}$ has finite first moment, i.e. for some (hence any) $z \in X$, and for any $x \in X$ one has $\int_{X} d(z, y) d m_{x}(y)<+\infty$ (see [43]).

A metric random walk space $[X, d, m]$ is a Polish metric space $(X, d)$ equipped with a random walk $m$.

Let $[X, d, m]$ be a metric random walk space. A Radon measure $\nu$ on $X$ is invariant for the random walk $m=\left(m_{x}\right)$ if

$$
d \nu(x)=\int_{X} d \nu(y) d m_{y}(x),
$$

that is, for any $\nu$-measurable set $A$, it holds that $A$ is $m_{x}$-measurable for $\nu$-almost all $x \in X, x \mapsto m_{x}(A)$ is $\nu$-measurable and

$$
\nu(A)=\int_{X} m_{x}(A) d \nu(x) .
$$

Hence, for any $f \in L^{1}(X, \nu)$, it holds that $f \in L^{1}\left(X, m_{x}\right)$ for $\nu$-a.e. $x \in X, x \mapsto \int_{X} f(y) d m_{x}(y)$ is $\nu$-measurable and

$$
\int_{X} f(x) d \nu(x)=\int_{X}\left(\int_{X} f(y) d m_{x}(y)\right) d \nu(x) .
$$

Note that, following the notation in the introduction, $\nu$ is invariant if $\nu * m=\nu$.

The measure $\nu$ is said to be reversible if, moreover, the detailed balance condition

$$
d m_{x}(y) d \nu(x)=d m_{y}(x) d \nu(y)
$$

holds. Under suitable assumptions on the metric random walk space $[X, d, m]$, such an invariant and reversible measure $\nu$ exists and is unique, as we will see below. Note that the reversibility condition implies the invariance condition.

We will assume that the measure space $(X, \nu)$ is $\sigma$-finite.

Example 1.2. (1) Let $\left(\mathbb{R}^{N}, d, \mathcal{L}^{N}\right)$, with $d$ the Euclidean distance and $\mathcal{L}^{N}$ the Lebesgue measure. Let $J: \mathbb{R}^{N} \rightarrow[0,+\infty$ [ be a measurable, nonnegative and radially symmetric function verifying $\int_{\mathbb{R}^{N}} J(z) d z=1$. In $\left(\mathbb{R}^{N}, d, \mathcal{L}^{N}\right)$ we have the following random walk,

$$
m_{x}^{J}(A):=\int_{A} J(x-y) d \mathcal{L}^{N}(y) \quad \text { for every Borel set } A \subset \mathbb{R}^{N} \text { and } x \in \mathbb{R}^{N} .
$$

Applying Fubini's Theorem it easy to see that the Lebesgue measure $\mathcal{L}^{N}$ is an invariant and reversible measure for this random walk. 
(2) Let $K: X \times X \rightarrow \mathbb{R}$ be a Markov kernel on a countable space $X$, i.e.,

$$
K(x, y) \geq 0 \quad \forall x, y \in X, \quad \sum_{y \in X} K(x, y)=1 \quad \forall x \in X .
$$

Then, for

$$
m_{x}^{K}(A):=\sum_{y \in A} K(x, y),
$$

$\left[X, d, m^{K}\right]$ is a metric random walk space for any metric $d$ on $X$. For irreducible and positive recurrent Markov chains (see for example [29]) there exists a unique stationary probability measure (also called steady state) on $X$, that is, a measure $\pi$ on $X$ satisfying

$$
\sum_{x \in X} \pi(x)=1 \quad \text { and } \quad \pi(y)=\sum_{x \in X} \pi(x) K(x, y) \quad \forall y \in X .
$$

This stationary probability measure $\pi$ is said to be reversible for $K$ if the following detailed balance equation

$$
K(x, y) \pi(x)=K(y, x) \pi(y)
$$

holds for $x, y \in X$. By Tonelli's Theorem for series, this balance condition is equivalent to the one given in (1.1) for $\nu=\pi$ :

$$
d m_{x}^{K}(y) d \pi(x)=d m_{y}^{K}(x) d \pi(y) .
$$

(3) A weighted discrete graph $G=(V(G), E(G))$ is a graph with vertex set $V(G)$ and edge set $E(G)$ such that to each edge $(x, y) \in E(G)$ (we will write $x \sim y$ if $(x, y) \in E(G)$ ) we assign a positive weight $w_{x y}=w_{y x}$. We consider that $w_{x y}=0$ if $(x, y) \notin E(G)$. We say that a vertex $x \in V(G)$ is simple if it has no loops, so that $w_{x x}=0$. A graph is said to be simple if all the vertices are simple.

A finite sequence $\left\{x_{k}\right\}_{k=0}^{n}$ of vertices on a graph is called a path if $x_{k} \sim x_{k+1}$ for all $k=$ $0,1, \ldots, n-1$. The length of a path is defined as the number, $n$, of edges in the path.

A graph $G=(V(G), E(G))$ is called connected if, for any two vertices $x, y \in V$, there is a path connecting $x$ and $y$, that is, a sequence of vertices $\left\{x_{k}\right\}_{k=0}^{n}$ such that $x_{0}=x$ and $x_{n}=y$. If $G=(V(G), E(G))$ is connected then define the graph distance $d_{G}(x, y)$ between any two distinct vertices $x, y$ as the minimum of the lengths of the paths connecting $x$ and $y$.

For each $x \in V(G)$ we define

$$
d_{x}:=\sum_{y \sim x} w_{x y} .
$$

When $w_{x y}=1$ for every $(x, y) \in E(G)$ with $x \sim y, d_{x}$ coincides with the degree of the vertex $x$ in the graph, that is, the number of edges containing $x$. A graph $G=(V(G), E(G))$ is called locally finite if each vertex belongs to a finite number of edges.

For each $x \in V(G)$ we define the following probability measure

$$
m_{x}^{G}=\frac{1}{d_{x}} \sum_{y \sim x} w_{x y} \delta_{y}
$$

If $G=(V(G), E(G))$ is a locally finite weighted connected graph, we have that $\left[V(G), d_{G},\left(m_{x}^{G}\right)\right]$ is a metric random walk space. Furthermore, it is not difficult to see that the measure $\nu_{G}$ defined as

$$
\nu_{G}(A):=\sum_{x \in A} d_{x}, \quad A \subset V(G),
$$

is an invariant and reversible measure for this random walk. 
(4) From a metric measure space $(X, d, \mu)$ we can obtain a metric random walk space, the so called $\epsilon$-step random walk associated to $\mu$, as follows. Assume that balls in $X$ have finite measure and that $\operatorname{Supp}(\mu)=X$. Given $\epsilon>0$, the $\epsilon$-step random walk on $X$, starting at point $x$, consists in randomly jumping in the ball of radius $\epsilon$ around $x$, with probability proportional to $\mu$; namely

$$
m_{x}^{\mu, \epsilon}:=\frac{\mu \mathrm{L} B(x, \epsilon)}{\mu(B(x, \epsilon))} .
$$

Note that $\mu$ is an invariant and reversible measure for the metric random walk space $\left[X, d, m^{\mu, \epsilon}\right]$.

(5) Given a metric random walk space $[X, d, m]$ with invariant and reversible measure $\nu$ for $m$, and given a $\nu$-measurable set $\Omega \subset X$ with $\nu(\Omega)>0$, if we define, for $x \in \Omega$,

$$
m_{x}^{\Omega}(A):=\int_{A} d m_{x}(y)+\left(\int_{X \backslash \Omega} d m_{x}(y)\right) \delta_{x}(A) \quad \text { for every Borel set } A \subset \Omega,
$$

we have that $\left[\Omega, d, m^{\Omega}\right]$ is a metric random walk space and it easy to see that $\nu\llcorner\Omega$ is reversible for $m^{\Omega}$.

Given a metric random walk space $[X, d, m]$, geometrically we may think of $m_{x}$ as a replacement for the notion of balls around $x$, while in probabilistic terms we can rather think of these data as defining a Markov chain whose transition probability from $x$ to $y$ in $n$ steps is

$$
d m_{x}^{* n}(y):=\int_{z \in X} d m_{z}(y) d m_{x}^{*(n-1)}(z)
$$

where $m_{x}^{* 1}=m_{x}$. Note that $m_{x}^{* n}=m_{x}^{*(n-1)} * m_{x}$ for any $x \in X$.

Observe that

$$
\int_{y \in X} f(y) d m_{x}^{* n}(y)=\int_{z \in X}\left(\int_{y \in X} f(y) d m_{z}(y)\right) d m_{x}^{*(n-1)}(z) .
$$

Thus, inductively,

$$
\int_{y \in X} d m_{x}^{* n}(y)=\int_{z \in X}\left(\int_{y \in X} d m_{z}(y)\right) d m_{x}^{*(n-1)}(z)=\int_{z \in X} d m_{x}^{*(n-1)}(z)=1 .
$$

Hence, $\left[X, d, m^{* n}\right]$ is also a metric random walk space. Moreover, if $\nu$ is invariant and reversible for $m$, then $\nu$ is also invariant and reversible for $m^{* n}$.

Definition 1.3. Let $[X, d, m]$ be a metric random walk space. We say that $[X, d, m]$ has the strong-Feller property if

$$
m_{x_{0}}(A)=\lim _{n \rightarrow+\infty} m_{x_{n}}(A) \quad \text { for every Borel set } A \subset X
$$

whenever $x_{n} \rightarrow x_{0}$ as $n \rightarrow+\infty$ in $(X, d)$.

Note that the examples of metric random walk spaces given in Example 1.2 have the strong-Feller property.

In [39] we study the concepts of $m$-perimeter and $m$-mean curvature associated with a metric random walk space $[X, d, m]$ with invariant and reversible measure $\nu$ with respect to $m$. For this aim, we introduce the notion of nonlocal interaction between two $\nu$-measurable subsets $A$ and $B$ of $X$ as

$$
L_{m}(A, B):=\int_{A} \int_{B} d m_{x}(y) d \nu(x) .
$$


For $L_{m}(A, B)<+\infty$, by the reversibility assumption on $\nu$, we have that

$$
L_{m}(A, B)=L_{m}(B, A) .
$$

We then define the concept of $m$-perimeter of a $\nu$-measurable subset $E \subset X$ as

$$
P_{m}(E)=L_{m}(E, X \backslash E)=\int_{E} \int_{X \backslash E} d m_{x}(y) d \nu(x) .
$$

If $\nu(E)<+\infty$, we have

$$
P_{m}(E)=\nu(E)-\int_{E} \int_{E} d m_{x}(y) d \nu(x) .
$$

It is easy to see that, on account of the reversibility of $\nu$,

$$
P_{m}(E)=\frac{1}{2} \int_{X} \int_{X}\left|\chi_{E}(y)-\chi_{E}(x)\right| d m_{x}(y) d \nu(x) .
$$
by

In the particular case of a graph $\left[V(G), d_{G}, m^{G}\right]$, the definition of perimeter of a set $E \subset V(G)$ is given

$$
|\partial E|:=\sum_{x \in E, y \in V \backslash E} w_{x y}
$$

Then, we have that

$$
|\partial E|=P_{m^{G}}(E) \text { for all } E \subset V(G)
$$

In [39], we also introduce the $m$-total variation of a function $u: X \rightarrow \mathbb{R}$ as

$$
T V_{m}(u):=\frac{1}{2} \int_{X} \int_{X}|u(y)-u(x)| d m_{x}(y) d \nu(x)
$$

and we prove the following Coarea formula. Note that

$$
P_{m}(E)=T V_{m}\left(\chi_{E}\right) \text {. }
$$

Theorem 1.4. ([39, Theorem 2.7]) For any $u \in L^{1}(X, \nu)$, let $E_{t}(u):=\{x \in X: u(x)>t\}$. Then

$$
T V_{m}(u)=\int_{-\infty}^{+\infty} P_{m}\left(E_{t}(u)\right) d t
$$

Let $E \subset X$ be $\nu$-measurable. For a point $x \in X$ we define the $m$-mean curvature of $\partial E$ at $x$ as

$$
\mathcal{H}_{\partial E}^{m}(x):=\int_{X}\left(\chi_{X \backslash E}(y)-\chi_{E}(y)\right) d m_{x}(y)=1-2 \int_{E} d m_{x}(y) .
$$

Note that $H_{\partial E}^{m}(x)$ can be computed for every $x \in X$, not only for points in $\partial E$. Furthermore, for a $\nu$-integrable set $E$,

$$
\int_{E} \mathcal{H}_{\partial E}^{m}(x) d \nu(x)=\int_{E}\left(1-2 \int_{E} d m_{x}(y)\right) d \nu(x)=\nu(E)-2 \int_{E} \int_{E} d m_{x}(y) d \nu(x),
$$

hence, having in mind (1.3), we obtain that

$$
\int_{E} \mathcal{H}_{\partial E}^{m}(x) d \nu(x)=2 P_{m}(E)-\nu(E)
$$


1.2. Ollivier-Ricci Curvature. Let $(X, d)$ be a Polish metric space and $\mathcal{M}^{+}(X)$ the set of positive Radon measures on $X$. Fix $\mu, \nu \in \mathcal{M}^{+}(X)$ satisfying the mass balance condition

$$
\mu(X)=\nu(X) .
$$

The Monge-Kantorovich problem is the minimization problem

$$
\min \left\{\int_{X \times X} d(x, y) d \gamma(x, y): \gamma \in \Pi(\mu, \nu)\right\},
$$

where $\Pi(\mu, \nu):=\left\{\right.$ Radon measures $\gamma$ in $\left.X \times X: \pi_{0} \# \gamma=\mu, \pi_{1} \# \gamma=\nu\right\}$, with $\pi_{\alpha}(x, y):=x+\alpha(y-x)$ for $\alpha \in\{0,1\}$.

For $1 \leq p<\infty$, the $p$-Wasserstein distance between $\mu, \nu$ is defined as

$$
W_{p}^{d}(\mu, \nu):=\left(\min \left\{\int_{X \times X} d(x, y)^{p} d \gamma(x, y): \gamma \in \Pi(\mu, \nu)\right\}\right)^{\frac{1}{p}} .
$$

The Monge-Kantorovich problem has a dual formulation that can be stated in this case as follows (see for instance [54, Theorem 1.14]).

Kantorovich-Rubinstein's Theorem. Let $\mu, \nu \in \mathcal{M}^{+}(X)$ be two measures satisfying the mass balance condition (1.6). Then,

$$
\begin{aligned}
W_{1}^{d}(\mu, \nu) & =\sup \left\{\int_{X} u d(\mu-\nu): u \in K_{d}(X)\right\} \\
& =\sup \left\{\int_{X} u d(\mu-\nu): u \in K_{d}(X) \cap L^{\infty}(X, \nu)\right\}
\end{aligned}
$$

where

$$
K_{d}(X):=\{u: X \mapsto \mathbb{R}:|u(y)-u(x)| \leq d(y, x)\} .
$$

In [43] Y. Ollivier gives the following definition of coarse Ricci curvature that we will call Ollivier-Ricci curvature.

Definition $1.5([43])$. On a given metric random walk space $[X, d, m]$, for any two distinct points $x, y \in X$, the Ollivier-Ricci curvature of $[X, d, m]$ along $(x, y)$ is defined as

$$
\kappa_{m}(x, y):=1-\frac{W_{1}^{d}\left(m_{x}, m_{y}\right)}{d(x, y)} .
$$

The Ollivier-Ricci curvature of $[X, d, m]$ is defined by

$$
\kappa_{m}:=\inf _{\substack{x, y \in X \\ x \neq y}} \kappa_{m}(x, y) .
$$

We will write $\kappa(x, y)$ instead of $\kappa_{m}(x, y)$, and $\kappa=\kappa_{m}$, if the context allows no confusion.

In the case that $(X, d, \mu)$ is a smooth complete Riemannian manifold, if $\left(m_{x}^{\mu, \epsilon}\right)$ is the $\epsilon$-step random walk associated to $\mu$ given in Example $1.2(4)$, then it is proved in [49] (see also [43]) that $\kappa_{m^{\mu, \epsilon}}(x, y)$ gives back the ordinary Ricci curvature when $\epsilon \rightarrow 0$, up to scaling by $\epsilon^{2}$. 
Example 1.6. Let $\left[\mathbb{R}^{N}, d, m^{J}\right]$ be the metric random walk space given in Example $1.2(1)$. Let us see that $\kappa(x, y)=0$. Given $x, y \in \mathbb{R}^{N}, x \neq y$, by Kantorovich-Rubinstein's Theorem, we have

$$
\begin{gathered}
W_{1}^{d}\left(m_{x}^{J}, m_{y}^{J}\right)=\sup \left\{\int_{\mathbb{R}^{N}} u(z)(J(x-z)-J(y-z)) d z: u \in K_{d}\left(\mathbb{R}^{N}\right)\right\} \\
=\sup \left\{\int_{\mathbb{R}^{N}}(u(x+z)-u(y+z)) J(z) d z: u \in K_{d}\left(\mathbb{R}^{N}\right)\right\} .
\end{gathered}
$$

Now, for $u \in K_{d}\left(\mathbb{R}^{N}\right)$, we have

$$
\int_{\mathbb{R}^{N}}(u(x+z)-u(y+z)) J(z) d z \leq\|x-y\| .
$$

Thus, $W_{1}^{d}\left(m_{x}^{J}, m_{y}^{J}\right) \leq\|x-y\|$. On the other hand, taking $u(z):=\frac{\langle z, x-y\rangle}{\|x-y\|}$, we have $u \in K_{d}\left(\mathbb{R}^{N}\right)$, hence

$$
W_{1}^{d}\left(m_{x}^{J}, m_{y}^{J}\right) \geq \int_{\mathbb{R}^{N}}(u(x+z)-u(y+z)) J(z) d z=\|x-y\| .
$$

Therefore,

$$
W_{1}^{d}\left(m_{x}^{J}, m_{y}^{J}\right)=\|x-y\|
$$

and, consequently, $\kappa(x, y)=0$.

Example 1.7. Let $\left[V(G), d_{G},\left(m_{x}^{G}\right)\right]$ be the metric random walk space associated to the locally finite weighted discrete graph $G=(V(G), E(G))$ given in Example $1.2(3)$ and let $N_{G}(x):=\{z \in V(G): z \sim x\}$ for $x \in V(G)$. Then, the Ollivier-Ricci curvature along $(x, y) \in E(G)$ is

$$
\kappa(x, y)=1-\frac{W_{1}^{d_{G}}\left(m_{x}, m_{y}\right)}{d_{G}(x, y)},
$$

where

$$
W_{1}^{d_{G}}\left(m_{x}, m_{y}\right)=\inf _{\mu \in \mathcal{A}} \sum_{z_{1} \sim x} \sum_{z_{2} \sim y} \mu\left(z_{1}, z_{2}\right) d_{G}\left(z_{1}, z_{2}\right),
$$

being $\mathcal{A}$ the set of all matrices with entries indexed by $N_{G}(x) \times N_{G}(y)$ such that $\mu\left(z_{1}, z_{2}\right) \geq 0$ and

$$
\sum_{z_{2} \sim y} \mu\left(z_{1}, z_{2}\right)=\frac{w_{x z_{1}}}{d_{x}}, \quad \sum_{z_{1} \sim x} \mu\left(z_{1}, z_{2}\right)=\frac{w_{y z_{2}}}{d_{y}}, \quad \text { for }\left(z_{1}, z_{2}\right) \in N_{G}(x) \times N_{G}(y) .
$$

There is an extensive literature about Ollivier-Ricci curvature on discrete graphs (see for instance, [8], [11], [18], [28], [30], [33], [43], [44], [45] and [46]).

\section{The Heat Flow on Metric Random Walk Spaces}

2.1. The Heat Flow. Let $[X, d, m]$ be a metric random walk space with invariant measure $\nu$ for $m$. For a function $u: X \rightarrow \mathbb{R}$ we define its nonlocal gradient $\nabla u: X \times X \rightarrow \mathbb{R}$ as

$$
\nabla u(x, y):=u(y)-u(x) \quad \forall x, y \in X,
$$

and for a function $\mathbf{z}: X \times X \rightarrow \mathbb{R}$, its $m$-divergence $\operatorname{div}_{m} \mathbf{z}: X \rightarrow \mathbb{R}$ is defined as

$$
\left(\operatorname{div}_{m} \mathbf{z}\right)(x):=\frac{1}{2} \int_{X}(\mathbf{z}(x, y)-\mathbf{z}(y, x)) d m_{x}(y) .
$$


The averaging operator on $[X, d, m]$ (see, for example, [43]) is defined as

$$
M_{m} f(x):=\int_{X} f(y) d m_{x}(y),
$$

when this expression has sense, and the Laplace operator as $\Delta_{m}:=M_{m}-I$, i.e.,

$$
\Delta_{m} f(x)=\int_{X} f(y) d m_{x}(y)-f(x)=\int_{X}(f(y)-f(x)) d m_{x}(y) .
$$

Note that

$$
\Delta_{m} f(x)=\operatorname{div}_{m}(\nabla f)(x)
$$

and $\left(M_{m}\right)^{n}=M_{m^{* n}}$ for $n \in \mathbb{N}$.

Due to the invariance of $\nu$ for the random walk $m$, both operators are well defined from $L^{1}(X, \nu)$ to $L^{1}(X, \nu),\left\|M_{m} f\right\|_{1} \leq\|f\|_{1}$ and $\left\|\Delta_{m} f\right\|_{1} \leq\|f\|_{1}$. Moreover, they map functions which are pointwise bounded by $C>0$ into functions pointwise bounded by $C$. Observe that the invariance of $\nu$ can be rewritten as the following property:

$$
\int_{X} \Delta_{m} f(x) d \nu(x)=0 \quad \forall f \in L^{1}(X, \nu) .
$$

In the case of the weighted discrete graph $G$ with the random walk defined in Example $1.2(3)$, the above operator is the graph Laplacian studied by many authors (see e.g. [10], [11], [22] or [30]).

By Jensen's inequality, we have that, for $f \in L^{2}(X, \nu) \cap L^{1}(X, \nu)$,

$$
\begin{gathered}
\left\|M_{m} f\right\|_{L^{2}(X, \nu)}^{2}=\int_{X}\left(\int_{X} f(y) d m_{x}(y)\right)^{2} d \nu(x) \\
\leq \int_{X} \int_{X} f^{2}(y) d m_{x}(y) d \nu(x)=\int_{X} f^{2}(x) d \nu(x)=\|f\|_{L^{2}(X, \nu)}^{2} .
\end{gathered}
$$

Therefore, $M_{m}$ and $\Delta_{m}$ are linear operators in $L^{2}(X, \nu)$ with domain

$$
D\left(M_{m}\right)=D\left(\Delta_{m}\right)=L^{2}(X, \nu) \cap L^{1}(X, \nu) .
$$

Moreover, in the case $\nu(X)<+\infty, M_{m}$ and $\Delta_{m}$ are bounded linear operators in $L^{2}(X, \nu)$ satisfying $\left\|M_{m}\right\| \leq 1$ and $\left\|\Delta_{m}\right\| \leq 2$.

If the invariant measure $\nu$ is reversible, the following integration by parts formula is straightforward:

$$
\int_{X} f(x) \Delta_{m} g(x) d \nu(x)=-\frac{1}{2} \int_{X \times X}(f(y)-f(x))(g(y)-g(x)) d m_{x}(y) d \nu(x)
$$

for $f, g \in L^{2}(X, \nu) \cap L^{1}(X, \nu)$.

In $L^{2}(X, \nu)$ we consider the symmetric form given by

$$
\mathcal{E}_{m}(f, g)=-\int_{X} f(x) \Delta_{m} g(x) d \nu(x)=\frac{1}{2} \int_{X \times X} \nabla f(x, y) \nabla g(x, y) d m_{x}(y) d \nu(x),
$$

with domain for both variables $D\left(\mathcal{E}_{m}\right)=L^{2}(X, \nu) \cap L^{1}(X, \nu)$, which is a linear and dense subspace of $L^{2}(X, \nu)$. 
Recall the definition of generalized product $\nu \otimes m_{x}$ (see, for instance, [1, Definition 2.2.7]), which is defined as the measure in $X \times X$ such that

$$
\int_{X \times X} g(x, y) d\left(\nu \otimes m_{x}\right)(x, y):=\int_{X}\left(\int_{X} g(x, y) d m_{x}(y)\right) d \nu(x)
$$

for every bounded Borel function $g$ with $\operatorname{supp}(g) \subset A \times B, A, B \subset \subset X$. In the previous definition we need to assume that the map $x \mapsto m_{x}(E)$ is $\nu$-measurable for any Borel set $E \in \mathcal{B}(X)$. Note that we can write

$$
\mathcal{E}_{m}(f, g)=\frac{1}{2} \int_{X \times X} \nabla f(x, y) \nabla g(x, y) d\left(\nu \otimes m_{x}\right)(x, y) .
$$

Theorem 2.1. Let $[X, d, m]$ be a metric random walk space with invariant and reversible measure $\nu$ for $m$. Then, $-\Delta_{m}$ is a non-negative self-adjoint operator in $L^{2}(X, \nu)$ with associated closed symmetric form $\mathcal{E}_{m}$, which, moreover, is a Markovian form.

Proof. For $f \in D\left(\Delta_{m}\right)$, by the integration by parts formula (2.2), we have

$$
\int_{X} f(x)\left(-\Delta_{m} f\right)(x) d \nu(x)=\mathcal{E}_{m}(f, f) \geq 0 .
$$

Also, as a consequence of (2.2), we have that $-\Delta_{m}$ is a self-adjoint operator in $L^{2}(X, \nu)$.

To prove the closedness of $\mathcal{E}_{m}$, consider $f_{n} \in D\left(\mathcal{E}_{m}\right)$ such that

$$
\mathcal{E}_{m}\left(f_{n}-f_{k}, f_{n}-f_{k}\right) \rightarrow 0, \quad \text { when } n, k \rightarrow+\infty,
$$

and

$$
\left\|f_{n}-f_{k}\right\|_{L^{2}(X, \nu)} \rightarrow 0, \quad \text { when } n, k \rightarrow+\infty .
$$

Since $f_{n} \rightarrow f$ in $L^{2}(X, \nu)$, we can assume that there exists a $\nu$-null set $N$ such that $f_{n}(x) \rightarrow f(x)$ for all $x \in X \backslash N$. Then, $\left(f_{n}(x)-f_{n}(y)\right)^{2} \rightarrow(f(x)-f(y))^{2}$ for all $(x, y) \in(X \backslash N) \times(X \backslash N)=$ $(X \times X) \backslash[(N \times X) \cup(X \times N)]$. Now, since $\nu$ is invariant, we have

$$
\begin{gathered}
\nu \otimes m_{x}([(N \times X) \cup(X \times N)])=\int_{N}\left(\int_{X} d m_{x}(y)\right) d \nu(x)+\int_{X}\left(\int_{X} \chi_{N}(y) d m_{x}(y)\right) d \nu(x) \\
=\nu(N)+\int_{X} \chi_{N}(y) d \nu(y)=2 \nu(N)=0 .
\end{gathered}
$$

Then, by Fatou's Lemma we have

$$
\begin{gathered}
\lim _{n \rightarrow \infty} \mathcal{E}_{m}\left(f_{n}-f, f_{n}-f\right)=\lim _{n \rightarrow+\infty} \frac{1}{2} \int_{X \times X}\left(\nabla\left(f_{n}-f\right)(x, y)\right)^{2} d\left(\nu \otimes m_{x}\right)(x, y) \\
=\lim _{n \rightarrow+\infty} \frac{1}{2} \int_{X \times X} \liminf _{k \rightarrow+\infty}\left(\nabla\left(f_{n}-f_{k}\right)(x, y)\right)^{2} d\left(\nu \otimes m_{x}\right)(x, y) \\
\leq \lim _{n \rightarrow+\infty} \liminf _{k \rightarrow+\infty} \frac{1}{2} \int_{X \times X}\left(\nabla\left(f_{n}-f_{k}\right)(x, y)\right)^{2} d\left(\nu \otimes m_{x}\right)(x, y)=0 .
\end{gathered}
$$

Therefore, $\mathcal{E}_{m}$ is closed. Moreover, for every 1-Lipschitz map $\eta: \mathbb{R} \rightarrow \mathbb{R}$ with $\eta(0)=0$, we have

$$
\mathcal{E}_{m}(\eta \circ f, \eta \circ f) \leq \mathcal{E}_{m}(f, f) \text { for every } f \in D\left(\mathcal{E}_{m}\right),
$$

and, hence, $\mathcal{E}_{m}$ satisfies the Markov property. 
By Theorem 2.1, as a consequence of the theory developed in [25, Chapter 1], we have that if $\left(T_{t}^{m}\right)_{t \geq 0}$ is the strongly continuous semigroup associated with $\mathcal{E}_{m}$, then $\left(T_{t}^{m}\right)_{t \geq 0}$ is a positivity preserving (i.e., $T_{t}^{m} f \geq 0$ if $f \geq 0$ ) Markovian semigroup (i.e., $0 \leq T_{t}^{m} f \leq 1$-a.e. whenever $f \in L^{2}(X, \nu), 0 \leq f \leq 1$ $\nu$-a.e.). Moreover, $\Delta_{m}$ is the infinitesimal generator of $\left(T_{t}^{m}\right)_{t \geq 0}$, that is

$$
\Delta_{m} f=\lim _{t \downarrow 0} \frac{T_{t}^{m} f-f}{t}, \quad \forall f \in D\left(\Delta_{m}\right) .
$$

From now on we denote $e^{t \Delta_{m}}:=T_{t}^{m}$ and we call $\left\{e^{t \Delta_{m}}: t \geq 0\right\}$ the heat flow on the metric random walk space $[X, d, m]$ with invariant and reversible measure $\nu$ for $m$. For every $u_{0} \in L^{2}(X, \nu)$, $u(t):=e^{t \Delta_{m}} u_{0}$ is the unique solution of the heat equation

$$
\left\{\begin{array}{l}
\frac{d u}{d t}(t)=\Delta_{m} u(t) \quad \text { for every } t \in(0,+\infty), \\
u(0)=u_{0},
\end{array}\right.
$$

in the sense that $u \in C\left([0,+\infty): L^{2}(X, \nu)\right) \cap C^{1}\left((0,+\infty): L^{2}(X, \nu)\right)$ and verifies (2.3), or equivalently,

$$
\left\{\begin{array}{l}
\frac{d u}{d t}(t, x)=\int_{X}(u(t)(y)-u(t)(x)) d m_{x}(y) \quad \text { for every } t>0 \text { and } \nu \text {-a.e. } x \in X, \\
u(0)=u_{0} .
\end{array}\right.
$$

By the Hille-Yosida exponential formula we have that

$$
e^{t \Delta_{m}} u_{0}=\lim _{n \rightarrow+\infty}\left[\left(I-\frac{t}{n} \Delta_{m}\right)^{-1}\right]^{n} u_{0} .
$$

As a consequence of $(2.1)$, if $\nu(X)<+\infty$, we have that the semigroup $\left(e^{t \Delta_{m}}\right)_{t \geq 0}$ conserves the mass. In fact

and, therefore,

$$
\frac{d}{d t} \int_{X} e^{t \Delta_{m}} u_{0}(x) d \nu(x)=\int_{X} \Delta_{m} u_{0}(x) d \nu(x)=0,
$$

$$
\int_{X} e^{t \Delta_{m}} u_{0}(x) d \nu(x)=\int_{X} u_{0}(x) d \nu(x) .
$$

Associated with $\mathcal{E}_{m}$ we define the energy functional

$$
\mathcal{H}_{m}(f):=\mathcal{E}_{m}(f, f),
$$

that is, $\mathcal{H}_{m}: L^{2}(X, \nu) \rightarrow[0,+\infty]$ is defined as

$$
\mathcal{H}_{m}(f):= \begin{cases}\frac{1}{2} \int_{X \times X}(f(x)-f(y))^{2} d m_{x}(y) d \nu(x) & \text { if } f \in L^{2}(X, \nu) \cap L^{1}(X, \nu), \\ +\infty & \text { else. }\end{cases}
$$

We denote

$$
D\left(\mathcal{H}_{m}\right):=L^{2}(X, \nu) \cap L^{1}(X, \nu) .
$$

Note that, for $f \in D\left(\mathcal{H}_{m}\right)$, we have

$$
\mathcal{H}_{m}(f)=-\int_{X} f(x) \Delta_{m} f(x) d \nu(x) .
$$


Remark 2.2. It is easy to see that the functional $\mathcal{H}_{m}$ is convex and, moreover, with a proof similar to the proof of closedness in Theorem 2.1, we get that the functional $\mathcal{H}_{m}$ is closed and lower semi-continuous in $L^{2}(X, \nu)$. Now, it is not difficult to see that $\partial \mathcal{H}_{m}=-\Delta_{m}$. Consequently, $-\Delta_{m}$ is a maximal monotone operator in $L^{2}(X, \nu)$. We can also consider the heat flow in $L^{1}(X, \nu)$. Indeed, if we define in $L^{1}(X, \nu)$ the operator $A$ as $A u=v \Longleftrightarrow v(x)=-\Delta_{m} u(x)$ for all $x \in X$, then $A$ is a completely accretive operator. In fact, let

$$
\mathcal{P}:=\left\{q \in C^{\infty}(\mathbb{R}): 0 \leq q^{\prime} \leq 1, \operatorname{supp}\left(q^{\prime}\right) \text { is compact and } 0 \notin \operatorname{supp}(q)\right\} .
$$

Given $f \in L^{1}(X, \nu)$, and $q \in \mathcal{P}$, applying (2.2), we have

$$
\int_{X} q(f(x)) A f(x) d \nu(x)=\frac{1}{2} \int_{X \times X}(q(f(y))-q(f(x)))(f(y)-f(x)) d m_{x}(y) d \nu(x) \geq 0 .
$$

Then, by [12, Proposition 2.2], we have that $A$ is a completely accretive operator. Moreover, $\overline{\partial \mathcal{H}}_{m}{ }^{1}(X, \nu)=$ $A$, thus $A$ is $m$-completely accretive in $L^{1}(X, \nu)$. Therefore, $A$ generates a $C_{0}$-semigroup $(S(t))_{t \geq 0}$ in $L^{1}(X, \nu)$ (see [16]) such that $S(t) f=e^{t \Delta_{m}} f$ for all $f \in L^{1}(X, \nu) \cap L^{2}(X, \nu)$, verifying

$$
\left\|S(t) u_{0}\right\|_{L^{p}(X, \nu)} \leq\left\|u_{0}\right\|_{L^{p}(X, \nu)} \quad \forall u_{0} \in L^{p}(X, \nu) \cap L^{1}(X, \nu), \quad 1 \leq p \leq+\infty .
$$

In the case that $\nu(X)<\infty$, we have that $S(t)$ is an extension to $L^{1}(X, \nu)$ of the heat flow $e^{t \Delta_{m}}$ in $L^{2}(X, \nu)$, that we will denote equally.

Example 2.3. (1) Consider the metric random walk space $\left[X, d, m^{K}\right]$ associated with the Markov kernel $K$ (see Example $1.2(2)$ ) and assume that the stationary probability measure $\pi$ is reversible. Then, the Laplacian $\Delta_{m^{K}}$ is given by

$$
\Delta_{m^{K}} f(x):=\int_{X} f(y) d m_{x}^{K}(y)-f(x)=\sum_{y \in X} K(x, y) f(y)-f(x) \quad \forall f \in L^{2}(X, \pi) .
$$

Consequently, given $u_{0} \in L^{2}(X, \pi), u(t):=e^{t \Delta_{m} K} u_{0}$ is the solution of the equation

$$
\left\{\begin{array}{l}
\frac{d u}{d t}(t, x)=\sum_{y \in X} K(x, y) u(t)(y)-u(t)(x) \quad \text { on }(0,+\infty), \\
u(0)=u_{0} .
\end{array}\right.
$$

Therefore, $e^{t \Delta_{m} K}=e^{t(K-I)}$ is the heat semigroup on $X$ with respect to the geometry determined by the Markov kernel $K$. In the case that $X$ is a finite set, we have

$$
e^{t \Delta_{m} K}=e^{t(K-I)}=e^{-t} \sum_{n=0}^{+\infty} \frac{t^{n} K^{n}}{n !} .
$$

(2) If we consider the metric random walk space $\left[\mathbb{R}^{N}, d, m^{J}\right]$, being $m^{J}=\left(m_{x}^{J}\right)$ the random walk defined in Example 1.2 (1), we have that, for the invariant measure $\nu=\mathcal{L}^{N}$, the Laplacian is given by

$$
\Delta_{m^{J}} f(x):=\int_{\mathbb{R}^{N}}(f(y)-f(x)) J(x-y) d y .
$$

Then, given $u_{0} \in L^{2}\left(\mathbb{R}^{N}, \mathcal{L}^{N}\right)$ we have that $u(t):=e^{t \Delta_{m^{J}}} u_{0}$ is the solution of the $J$-nonlocal heat equation

$$
\left\{\begin{array}{l}
\frac{d u}{d t}(t, x)=\int_{\mathbb{R}^{N}}(u(t)(y)-u(t)(x)) J(x-y) d y \text { in } \mathbb{R}^{N} \times(0,+\infty), \\
u(0)=u_{0}
\end{array}\right.
$$


In the case that $\Omega$ is a closed bounded subset of $\mathbb{R}^{N}$, if we consider the metric random walk space $\left[\Omega, d, m^{J, \Omega}\right]$, being $m^{J, \Omega}=\left(m^{J}\right)^{\Omega}$ (see Example $1.2(5)$ ), that is

$$
m_{x}^{J, \Omega}(A):=\int_{A} J(x-y) d y+\left(\int_{\mathbb{R}^{n} \backslash \Omega} J(x-z) d z\right) \delta_{x}(A) \quad \text { for every Borel set } A \subset \Omega,
$$

we have that

$$
\Delta_{m^{J, \Omega}} f(x)=\int_{\Omega}(f(y)-f(x)) d m_{x}^{J, \Omega}(y)=\int_{\Omega} J(x-y)(f(y)-f(x)) d y .
$$

Then we have that $u(t):=e^{t \Delta_{m} J, \Omega} u_{0}$ is the solution of the homogeneous Neumann problem for the $J$-nonlocal heat equation:

$$
\left\{\begin{array}{l}
\frac{d u}{d t}(t, x)=\int_{\Omega}(u(t)(y)-u(t)(x)) J(x-y) d x \text { in }(0,+\infty) \times \Omega, \\
u(0)=u_{0} .
\end{array}\right.
$$

See [4] for a comprehensive study of problems (2.6) and (2.7).

Observe that, in general, for a bounded set $\Omega \subset X$, and by using $m^{\Omega}$, we have that $u(t):=$ $e^{t \Delta_{m} \Omega} u_{0}$ is the solution of

$$
\left\{\begin{array}{l}
\frac{d u}{d t}(t, x)=\int_{\Omega}(u(t)(y)-u(t)(x)) d m_{x}(y) \text { in }(0,+\infty) \times \Omega, \\
u(0)=u_{0}
\end{array}\right.
$$

that, like (2.7), is an homogeneous Neumann problem for the $m$-heat equation.

In [38], it is shown, by means of the Fourier transform, that if $D \subset \mathbb{R}^{N}$ has $\mathcal{L}^{N}$-finite measure, then

$$
e^{\Delta_{m} J} \chi_{D}(x)=e^{-t} \sum_{n=0}^{\infty} \int_{D}(J *)^{n}(x-y) d y \frac{t^{n}}{n !} .
$$

In the next result we generalize (2.8) for general metric random walk spaces. We use the notation introduced in (1.2).

Theorem 2.4. Let $[X, d, m]$ be a metric random walk space with invariant and reversible measure $\nu$. Let $u_{0} \in L^{2}(X, \nu) \cap L^{1}(X, \nu)$. Then,

$$
e^{t \Delta_{m}} u_{0}(x)=e^{-t}\left(u_{0}(x)+\sum_{n=1}^{+\infty} \int_{X} u_{0}(y) d m_{x}^{* n}(y) \frac{t^{n}}{n !}\right)=e^{-t} \sum_{n=0}^{+\infty} \int_{X} u_{0}(y) d m_{x}^{* n}(y) \frac{t^{n}}{n !},
$$

where $\int_{X} u_{0}(y) d m_{x}^{* 0}(y)=u_{0}(x)$.

In particular, for $D \subset X$ with $\nu(D)<+\infty$, we have

$$
e^{t \Delta_{m}} \chi_{D}(x)=e^{-t}\left(\chi_{D}(x)+\sum_{n=1}^{+\infty} \int_{D} d m_{x}^{* n}(y) \frac{t^{n}}{n !}\right)=e^{-t} \sum_{n=0}^{+\infty} m_{x}^{* n}(D) \frac{t^{n}}{n !},
$$

where $m_{x}^{* 0}(D)=\chi_{D}(x)$. 
Proof. We define

$$
u(x, t)=e^{-t}\left(u_{0}(x)+\sum_{n=1}^{+\infty} \int_{X} u_{0}(y) d m_{x}^{* n}(y) \frac{t^{n}}{n !}\right) .
$$

Note that, since $u_{0} \in L^{1}(X, \nu)$, then $u_{0} \in L^{1}\left(X, m_{x}^{* n}\right)$ for $\nu$-a.e. $x \in X$ and every $n \in \mathbb{N}$, and

$$
\begin{aligned}
& \int_{X} \sum_{n=0}^{k}\left|\int_{X} u_{0}(y) d m_{x}^{* n}(y)\right| \frac{t^{n}}{n !} d \nu(x)=\sum_{n=0}^{k} \int_{X}\left|\int_{X} u_{0}(y) d m_{x}^{* n}(y)\right| \frac{t^{n}}{n !} d \nu(x) \\
\leq & \sum_{n=0}^{k} \int_{X} \int_{X}\left|u_{0}(y)\right| d m_{x}^{* n}(y) d \nu(x) \frac{t^{n}}{n !}=\sum_{n=0}^{k} \int_{X}\left|u_{0}(x)\right| d \nu(x) \frac{t^{n}}{n !} \leq e^{t}\left\|u_{0}\right\|_{L^{1}(X, \nu)} .
\end{aligned}
$$

Let

$$
f_{k}(x)=\sum_{n=0}^{k}\left|\int_{X} u_{0}(y) d m_{x}^{* n}(y)\right| \frac{t^{n}}{n !}
$$

then $0 \leq f_{k}(x) \leq f_{k+1}(x)<+\infty$ and $\int f_{k} d \nu \leq e^{t}\left\|u_{0}\right\|_{L^{1}(\nu)}$ for every $k \in \mathbb{N}$ so we may apply monotone convergence to get that

$$
\int_{X} \sum_{n=0}^{+\infty}\left|\int_{X} u_{0}(y) d m_{x}^{* n}(y)\right| \frac{t^{n}}{n !} d \nu(x) \leq e^{t}\left\|u_{0}\right\|_{L^{1}(X, \nu)},
$$

thus the function

$$
x \mapsto \sum_{n=0}^{+\infty}\left|\int_{X} u_{0}(y) d m_{x}^{* n}(y)\right| \frac{t^{n}}{n !}
$$

belongs to $L^{1}(X, \nu)$ and, consequently, is finite $\nu$-a.e. Note that the same is true for the function

$$
x \mapsto \sum_{n=0}^{+\infty} \int_{X}\left|u_{0}(y)\right| d m_{x}^{* n}(y) \frac{t^{n}}{n !} .
$$

From this we get that $u(x, t)$ is well defined and also the uniform convergence of the series for $t$ in compact subsets of $[0,+\infty)$. Hence,

$$
\frac{d u}{d t}(x, t)=-u(x, t)+e^{-t} \sum_{n=1}^{+\infty} \int_{X} u_{0}(y) d m_{x}^{* n}(y) \frac{t^{n-1}}{(n-1) !} .
$$

Therefore, to prove (2.9), we only need to show that

$$
e^{-t} \sum_{n=1}^{+\infty} \int_{X} u_{0}(y) d m_{x}^{* n}(y) \frac{t^{n-1}}{(n-1) !}=\int_{X} u(z, t) d m_{x}(z) .
$$

Now, by induction it is easy to see that

$$
\int_{X} u_{0}(y) d m_{x}^{* n}(y)=\int_{X}\left(\int_{X} u_{0}(y) d m_{z}^{*(n-1)}(y)\right) d m_{x}(z) .
$$

Thus,

$$
e^{-t} \sum_{n=1}^{+\infty} \int_{X} u_{0}(y) d m_{x}^{* n}(y) \frac{t^{n-1}}{(n-1) !}=e^{-t} \sum_{n=1}^{+\infty} \int_{X}\left(\int_{X} u_{0}(y) d m_{z}^{*(n-1)}(y)\right) d m_{x}(z) \frac{t^{n-1}}{(n-1) !}
$$




$$
=\int_{z \in X}\left(e^{-t} \sum_{n=1}^{+\infty} \int_{X} u_{0}(y) d m_{z}^{*(n-1)}(y) \frac{t^{n-1}}{(n-1) !}\right) d m_{x}(z)=\int_{X} u(z, t) d m_{x}(z),
$$

where we have interchanged the series and integral applying the dominated convergence Theorem because

$$
\left|e^{-t} \sum_{n=1}^{k} \int_{X} u_{0}(y) d m_{z}^{*(n-1)}(y) \frac{t^{n-1}}{(n-1) !}\right| \leq e^{-t} \sum_{n=1}^{+\infty} \int_{X}\left|u_{0}(y)\right| d m_{z}^{*(n-1)}(y) \frac{t^{n-1}}{(n-1) !}=: F(z)
$$

and $F$ belongs to $L^{1}(X, \nu)$, thus to $L^{1}\left(X, m_{x}\right)$ for $\nu$-a.e. $x \in X$.

2.2. Infinite Speed of Propagation and Ergodicity. In this section we study the infinite speed of propagation of the heat flow $\left(e^{t \Delta_{m}}\right)_{t \geq 0}$, that is, if it holds that

$$
e^{t \Delta_{m}} u_{0}>0 \text { for all } t>0 \text { whenever } 0 \leq u_{0} \in L^{2}(X, \nu), u_{0} \not \equiv 0 .
$$

We will see that this property is equivalent to a connectedness property of the space, to the ergodicity of the $m$-Laplacian $\Delta_{m}$ and to the ergodicity of the measure $\nu$.

Let $[X, d, m]$ be a metric random walk space with invariant measure $\nu$. For a $\nu$-measurable set $D$, we set

$$
N_{D}^{m}=\left\{x \in X: m_{x}^{* n}(D)=0, \forall n \in \mathbb{N}\right\}
$$

For $n \in \mathbb{N}$, we also define

$$
H_{D, n}^{m}=\left\{x \in X: m_{x}^{* n}(D)>0\right\}
$$

and

$$
H_{D}^{m}:=\bigcup_{n \in \mathbb{N}} H_{D, n}^{m}=\left\{x \in X: m_{x}^{* n}(D)>0 \text { for some } n \in \mathbb{N}\right\} .
$$

Note that $N_{D}^{m}$ and $H_{D}^{m}$ are disjoint and

$$
X=N_{D}^{m} \cup H_{D}^{m}
$$

Observe also that $N_{D}^{m}, H_{D, n}^{m}$ and $H_{D}^{m}$ are $\nu$-measurable.

Proposition 2.5. Let $[X, d, m]$ be a metric random walk space with invariant measure $\nu$. For a $\nu$ measurable set $D$, if $N_{D}^{m} \neq \emptyset$ then:

1.

$$
\begin{array}{ll}
m_{x}^{* n}\left(H_{D}^{m}\right)=0 & \text { for every } x \in N_{D}^{m} \text { and } n \in \mathbb{N}, \\
m_{x}^{* n}\left(N_{D}^{m}\right)=1 & \text { for every } x \in N_{D}^{m} \text { and } n \in \mathbb{N} .
\end{array}
$$

2. If $\nu(X)<+\infty$ or $\nu$ is reversible, then

$$
\begin{array}{ll}
m_{x}^{* n}\left(H_{D}^{m}\right)=1 \quad \text { for } \nu \text {-almost every } x \in H_{D}^{m}, \text { and for all } n \in \mathbb{N} . \\
m_{x}^{* n}\left(N_{D}^{m}\right)=0 \quad \text { for } \nu \text {-almost every } x \in H_{D}^{m}, \text { and for all } n \in \mathbb{N} .
\end{array}
$$

Consequently, for every $x \in N_{D}^{m}$ and $\nu$-a.e. $y \in H_{D}^{m}$ we have $m_{x} \perp m_{y}$, i.e. $m_{x}$ and $m_{y}$ are mutually singular. 
Proof. 1: Suppose that $m_{x}^{* k}\left(H_{D}^{m}\right)>0$ for some $x \in N_{D}^{m}$ and $k \in \mathbb{N}$, then, since $H_{D}^{m}=\cup_{n} H_{D, n}^{m}$ there exists $n \in \mathbb{N}$ such that $m_{x}^{* k}\left(H_{D, n}^{m}\right)>0$ but in that case we have

$$
m_{x}^{*(n+k)}(D)=\int_{z \in X} m_{z}^{* n}(D) d m_{x}^{* k}(z) \geq \int_{z \in H_{D, n}^{m}} m_{z}^{* n}(D) d m_{x}^{* k}(z)>0
$$

since $m_{z}^{* n}(D)>0$ for ever $z \in H_{D, n}^{m}$, and this contradicts that $x \in N_{D}^{m}$. The second statement in 1 . is then immediate.

2: Fix $n \in \mathbb{N}$. Using statement 1 . we have that for any finite $\nu$-measurable set $A$,

$$
\nu\left(A \cap H_{D}^{m}\right)=\int_{X} m_{x}^{* n}\left(A \cap H_{D}^{m}\right) d \nu(x)=\int_{H_{D}^{m}} m_{x}^{* n}\left(A \cap H_{D}^{m}\right) d \nu(x)
$$

because $m_{x}^{* n}\left(H_{D}^{m}\right)=0$ for every $x \in N_{D}^{m}$.

If $\nu\left(H_{D}^{m}\right)$ is finite, by $(2.11)$,

$$
\nu\left(H_{D}^{m}\right)=\int_{H_{D}^{m}} m_{x}^{* n}\left(H_{D}^{m}\right) d \nu(x) \quad \forall n \in \mathbb{N},
$$

and, therefore,

$$
m_{x}^{* n}\left(H_{D}^{m}\right)=1 \quad \text { for } \nu \text {-almost every } x \in H_{D}^{m} \text {, and for all } n \in \mathbb{N} \text {. }
$$

On the other hand, if $\nu\left(H_{D}^{m}\right)$ is not finite, since the space is $\sigma$-finite, we have that $H_{D}^{m}=\bigcup_{j=1}^{+\infty} H_{D}^{m} \cap B_{j}$, with $B_{j}$ open and $0<\nu\left(B_{j}\right)<+\infty$. Now, by $(2.11)$ and using reversibility of $\nu$,

$$
\nu\left(H_{D}^{m} \cap B_{j}\right)=\int_{H_{D}^{m}} m_{x}^{* n}\left(H_{D}^{m} \cap B_{j}\right) d \nu(x)=\int_{H_{m}^{D} \cap B_{j}} m_{x}^{* n}\left(H_{D}^{m}\right) d \nu(x) ;
$$

thus

Consequently,

$$
m_{x}^{* n}\left(H_{D}^{m}\right)=1 \quad \text { for } \nu \text {-almost every } x \in H_{m}^{D} \cap B_{j} .
$$

$$
m_{x}^{* n}\left(H_{D}^{m}\right)=1 \quad \text { for } \nu \text {-almost every } x \in H_{D}^{m} \text {, and for all } n \in \mathbb{N} \text {. }
$$

The second statement in 2 then follows.

Proposition 2.6. Let $[X, d, m]$ be a metric random walk space with invariant measure $\nu$ such that $\nu(X)<+\infty$ or $\nu$ is reversible. For a $\nu$-measurable set $D$, we have that, for every $n \in \mathbb{N}$ and for any finite $\nu$-measurable set $A$,

and

$$
\nu\left(A \cap H_{D}^{m}\right)=\int_{H_{D}^{m}} m_{x}^{* n}(A) d \nu(x),
$$

$$
\nu\left(A \cap N_{D}^{m}\right)=\int_{N_{D}^{m}} m_{x}^{* n}(A) d \nu(x) .
$$

Proof. If $N_{D}^{m}=\emptyset$ the result follows trivially, so let us suppose that $N_{D}^{m} \neq \emptyset$. By (2.11), and using Proposition 2.5, we have that, for any finite $\nu$-measurable set $A$,

$$
\nu\left(A \cap H_{D}^{m}\right)=\int_{H_{D}^{m}} m_{x}^{* n}\left(A \cap H_{D}^{m}\right) d \nu(x)=\int_{H_{D}^{m}} m_{x}^{* n}(A) d \nu(x)
$$


since

$$
m_{x}^{* n}(A)=m_{x}^{* n}\left(A \cap H_{D}^{m}\right)+m_{x}^{* n}\left(A \cap N_{D}^{m}\right)=m_{x}^{* n}\left(A \cap H_{D}^{m}\right)
$$

for $\nu$-a.e. $x \in H_{D}^{m}$ and every $n \in \mathbb{N}$. Similarly, one proves the other statement.

We have the following corollary.

Corollary 2.7. Let $[X, d, m]$ be a metric random walk space with invariant measure $\nu$ such that $\nu(X)<$ $+\infty$ or $\nu$ is reversible. For any $\nu$-measurable set $D$, we have that

$$
\nu\left(N_{D}^{m} \cap D\right)=0 .
$$

Consequently, if $\nu(D)>0$, then $D \subset H_{D}^{m}$ up to a $\nu$-null set; therefore, for $\nu$-a.e. $x \in D$ there exists $n=n(x) \in \mathbb{N}$ such that $m_{x}^{* n}(D)>0$.

Proof. If $D$ is finite, from Proposition 2.6,

$$
\nu\left(N_{D}^{m} \cap D\right)=\int_{N_{D}^{m}} m_{x}^{* n}(D) d \nu(x)=0 .
$$

If $D$ is not finite, then $D=\bigcup_{j=1}^{+\infty} D \cap B_{j}$, with $B_{j}$ open and $0<\nu\left(B_{j}\right)<+\infty$. Then,

$$
\nu\left(N_{D}^{m} \cap D\right) \leq \sum_{j=1}^{+\infty} \nu\left(N_{D}^{m} \cap D \cap B_{j}\right) .
$$

Now, from Proposition 2.6, $\nu\left(N_{D}^{m} \cap D \cap B_{j}\right)=\int_{N_{D}^{m}} m_{x}^{* n}\left(D \cap B_{j}\right) d \nu(x) \leq \int_{N_{D}^{m}} m_{x}^{* n}(D) d \nu(x)=0$, thus

$$
\nu\left(N_{D}^{m} \cap D\right)=0 .
$$

Definition 2.8. A metric random walk space $[X, d, m]$ with invariant measure $\nu$ is called random-walkconnected or m-connected if, for any $D \subset X$ with $0<\nu(D)<+\infty$, we have that $\nu\left(N_{D}^{m}\right)=0$.

A metric random walk space $[X, d, m]$ with invariant measure $\nu$ is called weakly-m-connected if, for any open set $D \subset X$ with $0<\nu(D)<+\infty$, we have that $\nu\left(N_{D}^{m}\right)=0$.

Theorem 2.9. Let $[X, d, m]$ be a metric random walk space with invariant and reversible measure $\nu$.

1. The space is m-connected if, and only if, for any non-null $0 \leq u_{0} \in L^{2}(X, \nu)$, we have $e^{t \Delta_{m}} u_{0}>0$ $\nu$-a.e. for all $t>0$.

2. The space is weakly-m-connected if, and only if, for any non-null $0 \leq u_{0} \in L^{2}(X, \nu) \cap C(X)$, we have $e^{t \Delta_{m}} u_{0}>0 \nu$-a.e. for all $t>0$.

Proof. $(1, \Rightarrow)$ : Given a non-null $0 \leq u_{0} \in L^{2}(X, \nu)$, there exist $D \subset X$ with $0<\nu(D)<+\infty$ and $\alpha>0$, such that $u_{0} \geq \alpha \chi_{D}$. Therefore, by Theorem 2.4,

$$
e^{t \Delta_{m}} u_{0}(x) \geq \alpha e^{t \Delta_{m}} \chi_{D}(x)=\alpha e^{-t} \sum_{n=0}^{\infty} m_{x}^{* n}(D) \frac{t^{n}}{n !}>0 \quad \text { for } \nu \text {-a.e. } x \in X .
$$

Indeed, if $x \in X \backslash N_{D}^{m}$ we have that $x \in H_{D}^{m}$, so there exists $n \in \mathbb{N}$ such that $m_{x}^{* n}(D)>0$. Then, since $\nu\left(N_{D}^{m}\right)=0$, we conclude. 
$(1, \Leftarrow)$ : Take $D \subset X$ with $0<\nu(D)<+\infty$, we have that

$$
e^{t \Delta_{m}} \chi_{D}(x)=e^{-t} \sum_{n=0}^{\infty} m_{x}^{* n}(D) \frac{t^{n}}{n !}>0 \quad \text { for } \nu \text {-a.e. } x \in X .
$$

Moreover, since $m_{x}^{* 0}=\delta_{x}$ and $m_{x}^{* n}(D)=0$ for every $x \in N_{D}^{m}$ and $n \geq 1$, we get

$$
e^{-t} \sum_{n=0}^{\infty} m_{x}^{* n}(D) \frac{t^{n}}{n !}=e^{-t} \chi_{D}(x) \quad \text { for } x \in N_{D}^{m},
$$

thus

Hence, by Corollary 2.7, $\nu\left(N_{D}^{m}\right)=0$.

$$
\chi_{D}(x)>0 \text { for } \nu \text {-a.e. } x \in N_{D}^{m} \text {. }
$$

The proof of $(2, \Rightarrow)$ is similar. $(\mathscr{2}, \Leftarrow)$ : Take $D \subset X$ open with $0<\nu(D)<+\infty$, since $\nu$ is regular there exists a compact set $K \subset D$ with $\nu(K)>0$. By Urysohn's lemma we may find a continuous function $0 \leq u_{0} \leq 1$ such that $u_{0}=0$ on $X \backslash D$ and $u_{0}=1$ on $K$, thus $u_{0} \leq \chi_{D}$. Hence

$$
e^{-t} \sum_{n=0}^{\infty} m_{x}^{* n}(D) \frac{t^{n}}{n !} \geq e^{t \Delta_{m}} u_{0}(x)>0 \quad \text { for } \nu \text {-a.e. } x \in X .
$$

So we conclude as before.

Remark 2.10. In the preceding proof, when $N_{D}^{m}=\emptyset$, we obtain that in fact

$$
e^{t \Delta_{m}} u_{0}(x)>0 \text { for all } x \in X \text { and for all } t>0 .
$$

We will say that the metric random walk space is strong $m$-connected when this happens for any non- $\nu$-null $0 \leq u_{0} \in L^{2}(X, \nu)$, and weakly strong $m$-connected if it holds for any non-null $0 \leq u_{0} \in L^{2}(X, \nu) \cap C(X)$.

The following result gives a characterization of $m$-connectedness in terms of the $m$-interaction of sets.

Proposition 2.11. Let $[X, d, m]$ be a metric random walk space with reversible measure $\nu$. The following statements are equivalent:

1. $X$ is m-connected.

2. If $A, B \subset X$ are $\nu$-measurable non- $\nu$-null sets such that $A \cup B=X$, then $L_{m}(A, B)>0$.

Proof. $1 \Rightarrow 2$ : Assume that $X$ is $m$-connected and let $A, B$ be as in statement 2. If

$$
0=L_{m}(A, B)=\int_{A} \int_{B} d m_{x}(y) d \nu(x),
$$

then

$$
m_{x}(B)=0 \text { for all } x \in A \backslash N_{1}, \nu\left(N_{1}\right)=0 .
$$

Now, since $\nu$ is invariant for $m$,

$$
0=\nu\left(N_{1}\right)=\int_{X} m_{x}\left(N_{1}\right) d \nu(x)
$$

and, consequently, there exists $N_{2} \subset X, \nu\left(N_{2}\right)=0$, such that

$$
m_{x}\left(N_{1}\right)=0 \quad \forall x \in X \backslash N_{2} .
$$


Hence, for $x \in A \backslash\left(N_{1} \cup N_{2}\right)$,

$$
\begin{aligned}
m_{x}^{* 2}(B) & =\int_{X} \chi_{B}(y) d m_{x}^{* 2}(y)=\int_{X}\left(\int_{X} \chi_{B}(y) d m_{z}(y)\right) d m_{x}(z) \\
& =\int_{X} m_{z}(B) d m_{x}(z)=\int_{A} m_{z}(B) d m_{x}(z)+\underbrace{\int_{B} m_{z}(B) d m_{x}(z)}_{=0, \text { since } x \in A \backslash N_{1}} \\
& =\underbrace{\int_{A \backslash N_{1}} m_{z}(B) d m_{x}(z)}_{=0, \text { since } z \in A \backslash N_{1}}+\underbrace{\int_{N_{1}} m_{z}(B) d m_{x}(z)}_{=0, \text { since } x \notin N_{2}}=0
\end{aligned}
$$

Working as above, we find $N_{3} \subset X, \nu\left(N_{3}\right)=0$, such that

$$
m_{x}\left(N_{1} \cup N_{2}\right)=0 \quad \forall x \in X \backslash N_{3} .
$$

Hence, for $x \in A \backslash\left(N_{1} \cup N_{2} \cup N_{3}\right)$, we have that

$$
\begin{aligned}
m_{x}^{* 3}(B) & =\int_{X} \chi_{B}(y) d m_{x}^{* 3}(y)=\int_{X}\left(\int_{X} \chi_{B}(y) d m_{z}^{* 2}(y)\right) d m_{x}(z) \\
& =\int_{X} m_{z}^{* 2}(B) d m_{x}(z) \leq \int_{A} m_{z}^{* 2}(B) d m_{x}(z)+\underbrace{\int_{B} m_{z}^{* 2}(B) d m_{x}(z)}_{=0, \text { since } x \in A \backslash\left(N_{1} \cup N_{2}\right)} \\
& \leq \underbrace{\int_{A \backslash\left(N_{1} \cup N_{2}\right)} m_{z}^{* 2}(B) d m_{x}(z)}_{=0, \text { since } z \in A \backslash\left(N_{1} \cup N_{2}\right)}+\underbrace{\int_{N_{1} \cup N_{2}} m_{z}^{* 2}(B) d m_{x}(z)}_{=0, \text { since } x \notin N_{3}}=0 .
\end{aligned}
$$

Inductively, we obtain that

$$
m_{x}^{* n}(B)=0 \quad \text { for } \nu \text {-a.e } x \in A \text { and every } n \in \mathbb{N} .
$$

Consequently,

$$
A \subset N_{B}^{m}
$$

up to a $\nu$-null set, which is a contradiction.

$2 \Rightarrow 1$ : Assume that statement 2 holds. If $X$ is not $m$-connected, then there exists a $\nu$-measurable set $D \subset X, 0<\nu(D)<+\infty$, such that $\nu\left(N_{D}^{m}\right)>0$. Moreover, by Corollary 2.7, $D \subset H_{D}^{m}$. Hence, we have that

$$
\nu\left(N_{D}^{m}\right)>0, \nu\left(H_{D}^{m}\right)>0 \text { and } X=N_{D}^{m} \cup H_{D}^{m},
$$

thus, by the hypothesis, $L_{m}\left(N_{D}^{m}, H_{D}^{m}\right)>0$, which is a contradiction.

Observe that the metric random walk space given in Example $1.2(1)$ is $m$-connected. This space has Ollivier-Ricci curvature equal to zero. In the next result we see that metric random walk spaces with positive Ollivier-Ricci curvature are $m$-connected. We will also see that connected graphs are always $m$-connected in Theorem 2.15 . 
Theorem 2.12. Let $[X, d, m]$ be a metric random walk space with finite invariant measure $\nu$. Assume that the Ollivier-Ricci curvature $\kappa$ satisfies $\kappa>0$. Then, $[X, d, m]$ with $\nu$ is $m$-connected and weakly strong m-connected.

Proof. Under the hypothesis $\kappa>-\infty$, recall that $\kappa \leq 1$ by definition, Y. Ollivier in [43, Proposition 20] proves the following $W_{1}$ contraction property:

Let $[X, d, m]$ be a metric random walk space. Then, for any two probability distributions, $\mu$ and $\mu^{\prime}$,

$$
W_{1}^{d}\left(\mu * m^{* n}, \mu^{\prime} * m^{* n}\right) \leq(1-\kappa)^{n} W_{1}^{d}\left(\mu, \mu^{\prime}\right) .
$$

Hence, under the hypothesis $\kappa>0$, Y. Ollivier in [43, Corollary 21] proves that the invariant measure $\nu$ (exists and) is unique up to a multiplicative constant, and that, for $\nu$ such that $\nu(X)=1$, the following hold:

$$
\begin{aligned}
& \text { (i) } \quad W_{1}^{d}\left(\mu * m^{* n}, \nu\right) \leq(1-\kappa)^{n} W_{1}^{d}(\mu, \nu) \quad \forall n \in \mathbb{N}, \forall \mu \in \mathcal{P}(X), \\
& \text { (ii) } \quad W_{1}^{d}\left(m_{x}^{* n}, \nu\right) \leq(1-\kappa)^{n} \frac{W_{1}^{d}\left(\delta_{x}, m_{x}\right)}{\kappa} \quad \forall n \in \mathbb{N}, \forall x \in X .
\end{aligned}
$$

So we will suppose, without loss of generality, that $\nu(X)=1$. By (2.13) and [55, Theorem 6.9], we have that

$$
\begin{aligned}
& \mu * m^{* n} \rightarrow \nu \text { weakly as measures, } \forall \mu \in \mathcal{P}(X), \\
& m_{x}^{* n} \rightarrow \nu \quad \text { weakly as measures, for every } x \in X .
\end{aligned}
$$

Let us now see that the space is $m$-connected if $\kappa>0$. Take $D \subset X$ with $0<\nu(D)<+\infty$ and suppose that $\nu\left(N_{D}^{m}\right)>0$. By Proposition 2.7, we have $\nu\left(H_{D}^{m}\right)>0$. Let

$$
\mu:=\frac{1}{\nu\left(H_{D}^{m}\right)} \nu\left\llcorner H_{D}^{m} \in \mathcal{P}(X)\right.
$$

and

$$
\mu^{\prime}:=\frac{1}{\nu\left(N_{D}^{m}\right)} \nu\left\llcorner N_{D}^{m} \in \mathcal{P}(X)\right.
$$

Now, by Proposition 2.6,

$$
\mu * m^{* n}=\mu
$$

and

$$
\mu^{\prime} * m^{* n}=\mu^{\prime}
$$

but then, by (2.12), we get

$$
W_{1}\left(\mu, \mu^{\prime}\right)=W_{1}\left(\mu * m^{* n}, \mu^{\prime} * m^{* n}\right) \leq(1-\kappa)^{n} W_{1}\left(\mu, \mu^{\prime}\right)
$$

which is only possible if $W_{1}\left(\mu, \mu^{\prime}\right)=0$ since $1-\kappa<1$. Hence,

$$
\mu=\mu^{\prime} \text {, }
$$

and this implies $1=\mu^{\prime}\left(N_{D}^{m}\right)=\mu\left(N_{D}^{m}\right)=0$ which gives a contradiction. Therefore, $\nu\left(N_{D}^{m}\right)=0$ so the space is $m$-connected.

If $D$ is open and $\nu(D)>0$ then $N_{D}^{m}=\emptyset$. Indeed, for $x \in N_{D}^{m}$, by (2.14), we have

$$
0<\nu(D) \leq \liminf _{n} m_{x}^{* n}(D)=0
$$


Remark 2.13. In [29], it is shown that uniqueness of the invariant probability measure implies its ergodicity. Consequently, Theorem 2.12 follows from [43, Corollary 21] (see Theorem 2.19 for the relation between ergodicity and $m$-connectedness). We have presented the result for the sake of completeness and using the framework of $m$-connectedness.

Note that, in the previous result, a condition involving the random walk and the metric on the ambient space yields the $m$-connectedness of the metric random walk space, which is, a priori, unrelated to the metric.

Proposition 2.14. Let $[X, d, m]$ be a metric random walk space with invariant measure $\nu$ such that supp $\nu=X$ and either $\nu(X)<+\infty$, or $\nu$ is reversible. Suppose further that $[X, d, m]$ has the strongFeller property and $(X, d)$ is connected, then $[X, d, m]$ with $\nu$ is strong $m$-connected.

Proof. Recall that, since $[X, d, m]$ has the strong-Feller property, $\left[X, d, m^{* k}\right]$ also has this property for any $k \in \mathbb{N}$. Take $D$ with $0<\nu(D)<+\infty$. Let us see first that $H_{D}^{m}$ is open or, equivalently, that $N_{D}^{m}$ is closed; indeed, if we have $\left(x_{n}\right) \subset N_{D}^{m}$ such that $\lim _{n \rightarrow \infty} x_{n}=x \in X$ then

$$
m_{x}^{* k}(D)=\lim _{n \rightarrow \infty} m_{x_{n}}^{* k}(D)=0
$$

for any $k \in \mathbb{N}$, thus $x \in N_{D}^{m}$.

On the other hand, if $m_{x}\left(H_{D}^{m}\right)<1$ for some $x \in H_{D}^{m}$, since $[X, d, m]$ has the strong-Feller property, there exists $r>0$ such that $m_{y}\left(H_{D}^{m}\right)<1$ for every $y \in B_{r}(x) \subset H_{D}^{m}$. Therefore, by $(2.10), \nu\left(B_{r}(x)\right)=0$, which is a contradiction since $\operatorname{supp} \nu=X$. Hence,

$$
m_{x}\left(H_{D}^{m}\right)=1 \text { if, and only if, } \quad x \in H_{D}^{m} .
$$

Then, given $\left(x_{n}\right) \subset H_{D}^{m}$ such that $\lim _{n \rightarrow \infty} x_{n}=x \in X$, we have

$$
m_{x}\left(H_{D}^{m}\right)=\lim _{n \rightarrow \infty} m_{x_{n}}\left(H_{D}^{m}\right)=1,
$$

so $x \in H_{D}^{m}$. Therefore, $H_{D}^{m}$ is also closed and then, since $X$ is connected, we have that $X=H_{D}^{m}$, which implies that $N_{D}^{m}=\emptyset$.

Theorem 2.15. Let $\left[V(G), d_{G},\left(m_{x}^{G}\right)\right]$ be the metric random walk space associated with the locally finite weighted connected graph $G=(V(G), E(G))$. Then $\left[V(G), d_{G}, m^{G}\right]$ with $\nu_{G}$ is strong $m$-connected.

Proof. Take $D \subset V(G)$ with $\nu_{G}(D)>0$, and let us see that $N_{D}^{m^{G}}=\emptyset$. Suppose that there exists $y \in N_{D}^{m^{G}}$, this implies that

$$
\left(m^{G}\right)_{y}^{* n}(D)=0 \quad \forall n \in \mathbb{N} .
$$

Now, given $x \in D$, there is a path of length $m,\left\{x, z_{1}, z_{2}, \ldots, z_{m-1}, y\right\}, x \sim z_{1} \sim z_{2} \sim \cdots \sim z_{m-1} \sim y$, and then

$$
\left(m^{G}\right)_{y}^{* n}(\{x\}) \geq \frac{w_{y z_{m-1}} w_{z_{m-1} z_{m-2}} \cdots w_{z_{2} z_{1}} w_{z_{1} x}}{d_{y} d_{z_{m-1}} d_{z_{m-2}} \cdots d_{z_{2}} d_{z_{1}}}>0
$$

which is in contradiction with (2.15).

The next examples show that there is no relation between $m$-connectedness and classical connectedness, i.e., there are connected metric random walk spaces that are not $m$-connected and, conversely, there are $m$-connected metric random walk spaces that are not connected. 
Example 2.16. Take $([0,1], d)$ with $d$ the Euclidean distance and let $C$ be the Cantor set. Let $\mu$ be the Cantor distribution, that is, the probability measure whose cumulative distribution function $F(x)=$ $\mu([0, x))$ is the Cantor function. We have that $\mu$ is singular with respect to the Lebesgue measure and its support is the Cantor set. We denote $\eta:=\mathcal{L}^{1}\llcorner[0,1]$ and define the random walk

$$
m_{x}:= \begin{cases}\eta & \text { if } x \in[0,1] \backslash C, \\ \mu & \text { if } x \in C .\end{cases}
$$

Then, $\nu=\eta+\mu$ is invariant and reversible. Indeed,

$$
\begin{gathered}
\int_{(0,1)} \int_{(0,1)} f(y) d m_{x}(y) d \nu(x)=\int_{(0,1) \backslash C} \int_{(0,1)} f(y) d y d x+\int_{C} \int_{C} f(y) d \mu(y) d \mu(x)= \\
=\int_{(0,1)} f(y) d y+\int_{C} f(y) d \mu(y)=\int_{(0,1)} f(y) d \nu(y),
\end{gathered}
$$

Similarly, we prove that $\nu$ is reversible.

On the other hand, $m_{x}^{* n}=m_{x}$ for any $x \in(0,1)$ and $n \in \mathbb{N}$. In fact, if $x \in C$, we have

$$
\begin{gathered}
\int_{y \in X} f(y) d m_{x}^{* 2}(y)=\int_{z \in(0,1)}\left(\int_{y \in 0,1)} f(y) d m_{z}(y)\right) d m_{x}(z)=\int_{z \in(0,1)}\left(\int_{y \in(0,1)} f(y) d m_{z}(y)\right) d \mu(z) \\
=\int_{z \in C}\left(\int_{y \in(0,1)} f(y) d m_{z}(y)\right) d \mu(z)=\int_{z \in C}\left(\int_{y \in C} f(y) d \mu(y)\right) d \mu(z)=\int_{X} f(y) d m_{x}(y),
\end{gathered}
$$

and the proof for $x \in(0,1) \backslash C$ is similar.

Consequently, $m_{x}^{* n}(C)=0$ for every $x \in(0,1) \backslash C$ and for every $n \in \mathbb{N}$, so that $\nu\left(N_{C}^{m}\right) \geq \nu((0,1) \backslash C)=$ $1>0$ and, therefore, the space $[(0,1), d, m]$ is not $m$-connected.

For $x, y \in(0,1), x \neq y$, if $x, y \in C$, or $x, y \in(0,1) \backslash C$, then $W_{1}\left(m_{x}, m_{y}\right)=0$ and hence $\kappa(x, y)=1$; otherwise, if $x \in C$ and $y \in(0,1) \backslash C$ then $W_{1}\left(m_{x}, m_{y}\right)=W_{1}(\mu, \eta)$. Hence $\kappa(x, y)=1-\frac{W_{1}(\mu, \eta)}{|x-y|}$. Consequently, since $\kappa=\inf _{x \neq y} \kappa(x, y)$ and $\kappa(x, y)=1$ if $x, y \in C$ or $x, y \in(0,1) \backslash C$, we get

$$
\kappa=\inf _{x \in C, y \notin C}\left(1-\frac{W_{1}(\mu, \eta)}{|x-y|}\right)=-\infty .
$$

Example 2.17. Let $\Omega:=(]-\infty, 0] \cup\left[\frac{1}{2},+\infty[) \times \mathbb{R}^{N-1}\right.$ and consider the metric random walk space $\left[\Omega, d, m^{J, \Omega}\right]$, with $d$ the Euclidean distance and $J(x)=\frac{1}{\left|B_{1}(0)\right|} \chi_{B_{1}(0)}$ (see Example (1.2) (1)). It is easy to see that this space with reversible and invariant measure $\nu=\mathcal{L} L \Omega$ is $m$-connected, but $(\Omega, d)$ is not connected. Let us see that its Ollivier-Ricci curvature $\kappa$ is negative. Indeed, take $x=\left(-\frac{1}{2}, 0, \ldots, 0\right) \in \Omega$, and $y=(2,0, \ldots, 0) \in \Omega$. Then, we have that $u\left(x_{1}, x_{2}, \ldots x_{N}\right)=-x_{1}$ is a Kantorovich potential for the 
transport of $m_{x}^{J}$ to $m_{y}^{J}$, consequently

$$
\begin{aligned}
W_{1}\left(m_{x}^{J, \Omega}, m_{y}^{J, \Omega}\right) & \geq \int_{\Omega} u(z)\left(d m_{x}^{J, \Omega}(z)-d m_{y}^{J, \Omega}(z)\right) \\
& =\int_{\Omega} u(z)\left(d m_{x}^{J}(z)-d m_{y}^{J}(z)\right)+\left(\int_{\mathbb{R}^{N} \backslash \Omega} d m_{x}^{J}(z)\right) u(x) \\
& =\int_{\mathbb{R}^{N}} u(z)\left(d m_{x}^{J}(z)-d m_{y}^{J}(z)\right)+\int_{\mathbb{R}^{N} \backslash \Omega}(u(x)-u(z)) d m_{x}^{J}(z) \\
& =W_{1}\left(m_{x}^{J}, m_{y}^{J}\right)+\int_{\mathbb{R}^{N} \backslash \Omega}\left(\frac{1}{2}+z_{1}\right) d m_{x}^{J}(z) \\
& >d(x, y) .
\end{aligned}
$$

Therefore, the Ollivier-Ricci curvature of $\left[\Omega, d, m^{J, \Omega}\right]$

$$
\kappa \leq \kappa(x, y)=1-\frac{W_{1}\left(m_{x}^{J, \Omega}, m_{y}^{J, \Omega}\right)}{d(x, y)}<0 .
$$

For $\Omega=(]-\infty, 0] \cup\left[2,+\infty[) \times \mathbb{R}^{N-1}\right.$, neither $\left[\Omega, d, m^{J, \Omega}\right]$ with $\nu=\mathcal{L} L \Omega$ is $m$-connected, nor $(\Omega, d)$ is connected. As above, we can prove that its Ollivier-Ricci curvature is negative.

In a similar way we have that, for $\Omega=\mathbb{R}^{N} \backslash(0,2)^{N},\left[\Omega, d, m^{J, \Omega}\right]$ with $\nu=\mathcal{L}\llcorner\Omega$ is $m$-connected, $(\Omega, d)$ is connected and its Ollivier-Ricci curvature is $\kappa<0$.

We will now relate the $m$-connectedness property with other known concepts in the literature. Let us begin with the concept of ergodicity (see, for example, [29]).

Definition 2.18. Let $[X, d, m]$ be a metric random walk space with invariant probability measure $\nu$. A Borel set $B \subset X$ is said to be invariant with respect to the random walk $m$ if $m_{x}(B)=1$ whenever $x$ is in $B$.

The invariant probability measure $\nu$ is said to be ergodic if $\nu(B)=0$ or $\nu(B)=1$ for every invariant set $B$ with respect to the random walk $m$.

Theorem 2.19. Let $[X, d, m]$ be a metric random walk space with invariant probability measure $\nu$. Then, the following assertions are equivalent:

(i) $[X, d, m]$ with $\nu$ is m-connected.

(ii) $\nu$ is ergodic.

Proof. $(i) \Rightarrow(i i)$. If $\nu$ is not ergodic, there exists an invariant set $B$ with respect to the random walk $m$ such that $0<\nu(B)<1$. However, note that $B$ is also invariant with respect to $m^{* 2}$. Indeed,

$$
m_{x}^{* 2}(B)=\int_{X} m_{z}(B) d m_{x}(z) \geq \int_{B} m_{z}(B) d m_{x}(z)=m_{x}(B)=1
$$


for every $x \in B$. Inductively, we obtain that, in fact, $B$ is invariant for $m^{* n}$, for every $n \in \mathbb{N}$. Therefore, since

$$
\nu(B)=\int_{X} m_{x}^{* n}(B) d \nu(x) \geq \int_{B} m_{x}^{* n}(B) d \nu(x)=\nu(B) \quad \text { for every } n \in \mathbb{N},
$$

we obtain that $m_{x}^{* n}(B)=0$ for every $n \in \mathbb{N}$ and $\nu$-a.e. $x \in X \backslash B$. Therefore, $X \backslash B \subset N_{B}^{m} \nu$-a.e., thus $\nu\left(N_{B}^{m}\right)>0$ and, consequently, $[X, d, m]$ with $\nu$ is not $m$-connected.

$($ ii $) \Rightarrow(i)$. Let $D \subset X$ be a $\nu$-measurable set with $\nu(D)>0$. By Proposition 2.5 we have that $N_{D}^{m}$ is invariant with respect to the random walk $m$. Then, since $\nu$ is ergodic, we have that $\nu\left(N_{D}^{m}\right)=0$ or $\nu\left(N_{D}^{m}\right)=1$. Now, since $\nu(D)>0$, by Corollary 2.7, we have that $\nu\left(N_{D}^{m}\right)=0$ and, consequently, $[X, d, m]$ with $\nu$ is $m$-connected.

Following Bakry, Gentil and Ledoux [6], we give the following definition.

Definition 2.20. Let $[X, d, m]$ be a metric random walk space with invariant measure $\nu$. We say that $\Delta_{m}$ is ergodic if, for $u \in \operatorname{Dom}\left(\Delta_{m}\right), \Delta_{m} u=0$ implies that $u$ is constant (being this constant 0 if $\nu$ is not finite).

Theorem 2.21. Let $[X, d, m]$ be a metric random walk space with finite invariant measure $\nu$. Then,

$\Delta_{m}$ is ergodic $\Leftrightarrow[X, d, m]$ is random walk connected.

Proof. $(\Rightarrow)$ : Suppose that $\nu(X)<+\infty$ and $[X, d, m]$ is not $m$-connected, then there exists $D \subset X$ with $\nu(D)>0$ such that $\nu\left(N_{D}^{m}\right)>0$. Recall that $\nu\left(H_{D}^{m}\right)>0$. Consider the function

$$
u(x)=\chi_{H_{D}^{m}}(x),
$$

and note that $u \in L^{2}(X, \nu)$ since $\nu$ is finite. Now,

$$
\Delta_{m} u(x)=\int_{X}\left(\chi_{H_{D}^{m}}(y)-\chi_{H_{D}^{m}}(x)\right) d m_{x}(y)=m_{x}\left(H_{D}^{m}\right)-\chi_{H_{D}^{m}}(x),
$$

hence, by Proposition 2.5,

$$
\Delta_{m} u=0 \quad \nu \text {-a.e. }
$$

but $u$ is not equal to a constant $\nu$-a.e., and, consequently, $\Delta_{m}$ is not ergodic. 
$(\Leftarrow)$ : Suppose now that $[X, d, m]$ is $m$-connected and that there exists $u \in L^{2}(X, \nu)$ such that $\Delta_{m} u=0$ $\nu$-a.e. but $u$ is not $\nu$-a.e. equal to a constant function. Then, we may find $U, V \subset X$ with positive $\nu$ measure such that $u(x)<u(y)$ for every $x \in U$ and $y \in V$. Note that

$$
\begin{aligned}
\Delta_{m^{* n}} u(x) & =\int_{X}(u(y)-u(x)) d m_{x}^{* n}(y) \\
& =\int_{X} \int_{X}(u(z)-u(x)) d m_{y}(z) d m_{x}^{*(n-1)}(y) \\
& =\int_{X} \int_{X}(u(z)-u(y)) d m_{y}(z) d m_{x}^{*(n-1)}(y)+\int_{X} \int_{X}(u(y)-u(x)) d m_{y}(z) d m_{x}^{*(n-1)}(y) \\
& =\int_{X} \Delta_{m} u(y) d m_{x}^{*(n-1)}(y)+\int_{X}(u(y)-u(x)) d m_{x}^{*(n-1)}(y) \\
& =\int_{X} \Delta_{m} u(y) d m_{x}^{*(n-1)}(y)+\Delta_{m^{*(n-1)}} u(x),
\end{aligned}
$$

thus

$$
\left|\Delta_{m^{* n}} u(x)\right| \leq \int_{X}\left|\Delta_{m} u(y)\right| d m_{x}^{*(n-1)}(y)+\left|\Delta_{m^{*(n-1)}} u(x)\right| .
$$

Now, using the invariance of $\nu$,

$$
\int_{X} \int_{X}\left|\Delta_{m} u(y)\right| d m_{x}^{*(n-1)}(y) d \nu(x)=\int_{X}\left|\Delta_{m} u(x)\right| d \nu(x)=0
$$

so

$$
\int_{X}\left|\Delta_{m} u(y)\right| d m_{x}^{*(n-1)}(y)=0 \quad \text { for } \nu \text {-a.e. } x \in X,
$$

thus, by induction on (2.16), $\Delta_{m^{* n}} u(x)=0$ for $\nu$-a.e. $x \in X$ and every $n \in \mathbb{N}$. Since $[X, d, m]$ is $m$-connected we have $\nu\left(N_{V}^{m}\right)=\nu\left(X \backslash H_{V}^{m}\right)=0$, so there exists $n \in \mathbb{N}$ such that $\nu\left(U \cap H_{V, n}^{m}\right)>0$. Consequently, we get a contradiction:

$$
0=\mathcal{H}_{m^{* n}}(u)=\int_{X} \int_{X} \nabla u(x, y)^{2} d m_{x}^{* n}(y) d \nu(x) \geq \int_{U \cap H_{V, n}^{m}} \int_{V} \nabla u(x, y)^{2} d m_{x}^{* n}(y) d \nu(x)>0 .
$$

Let $[X, d, m]$ be a metric random walk space with invariant and reversible measure $\nu$. It is easy to see that $\Delta_{m}$ is ergodic if, and only if, $e^{t \Delta_{m}} f=f$ for all $t \geq 0$ implies that $f$ is constant. Moreover, we have the following result.

Proposition 2.22. Let $[X, d, m]$ be a metric random walk space with invariant and reversible measure $\nu$. For every $f \in L^{2}(X, \nu)$,

$$
\lim _{t \rightarrow \infty} e^{t \Delta_{m}} f=f_{\infty} \in\left\{u \in L^{2}(X, \nu): \Delta_{m} u=0\right\} .
$$

Suppose further that $\Delta_{m}$ is ergodic:

(i) If $\nu(X)=+\infty$, then $f_{\infty}=0$.

(ii) If $\nu(X)<+\infty$, then $f_{\infty}=\frac{1}{\nu(X)} \int_{X} f(x) d \nu(x)$. 
Proof. The first result follows from [15, Theorem 3.11]. The second part is a consequence of the ergodicity of $\Delta_{m}$ and the conservation of mass (2.4).

When the invariant measure is a probability measure, the relation between both concepts of ergodicity, the one for the invariant measure and the one for the Laplacian was known; see, for example, [29]. Let us now give another characterization of the ergodicity in terms of geometric properties.

Lemma 2.23. Let $[X, d, m]$ be a metric random walk space with invariant and reversible measure $\nu$ and assume that $\nu(X)<+\infty$. Then for every $\nu$-measurable subset $D \subset X$ we have

$$
\Delta_{m} \chi_{D}(x)=0 \quad \Leftrightarrow \quad m_{x}(D)=\chi_{D}(x),
$$

and

$$
\Delta_{m} \chi_{D}=0 \quad \Leftrightarrow \quad P_{m}(D)=0 \quad \Leftrightarrow \quad \int_{D} \mathcal{H}_{\partial D}^{m}(x) d \nu(x)=-\nu(D) .
$$

Proof. Since

$$
\Delta_{m} \chi_{D}(x)=\int_{X}\left(\chi_{D}(y)-\chi_{D}(x)\right) d m_{x}(y)=m_{x}(D)-\chi_{D}(x)
$$

we have that $\Delta_{m} \chi_{D}(x)=0$ if, and only if, $\chi_{D}(x)-m_{x}(D)=0$, and we get (2.17).

Suppose now that $\Delta_{m} \chi_{D}=0$, then $\chi_{D}(x)=m_{x}(D)$, thus integrating this expression over $D$ with respect to $\nu$, we get

$$
P_{m}(D)=\nu(D)-\int_{D} \int_{D} d m_{x}(y) d \nu(x)=0 .
$$

Conversely, if $P_{m}(D)=0$, we have

$$
\nu(D)=\int_{D} m_{x}(D) d \nu(x)
$$

Then, on one hand,

$$
m_{x}(D)=\chi_{D}(x) \text { for } \nu \text {-a.e. } x \in D,
$$

and, on the other hand, since

$$
\nu(D)=\int_{X} m_{x}(D) d \nu(x)=\int_{D} m_{x}(D) d \nu(x)+\int_{X \backslash D} m_{x}(D) d \nu(x),
$$

we get

$$
\int_{X \backslash D} m_{x}(D) d \nu(x)=0
$$

thus

$$
m_{x}(D)=\chi_{D}(x) \text { for } \nu \text {-a.e. } x \in X \backslash D \text {. }
$$

Therefore,

$$
m_{x}(D)=\chi_{D}(x) \text { for } \nu \text {-a.e. } x \in X
$$

and, by (2.17), we get $\Delta_{m} \chi_{D}=0$.

For the second equivalence, by (1.5), we have that

$$
\int_{D} \mathcal{H}_{\partial D}^{m}(x) d \nu(x)=2 P_{m}(D)-\nu(D),
$$

thus $P_{m}(D)=0$ if, and only if, $\int_{D} \mathcal{H}_{\partial D}^{m}(x) d \nu(x)=-\nu(D)$. 
Theorem 2.24. Let $[X, d, m]$ be a metric random walk space with invariant and reversible measure $\nu$ and assume that $\nu(X)<+\infty$. The following facts are equivalent:

(1) $\Delta_{m}$ is ergodic;

(2) $\Delta_{m} \chi_{D}=0$ for a $\nu$-measurable set $D$ implies that $\chi_{D}$ is constant;

(3) $P_{m}(D)>0$ for every $\nu$-measurable set $D$ such that $0<\nu(D)<\nu(X)$;

(4) The $\nu$-mean value of the $m$-mean curvature of $\partial D$ in $D$ satisfies

$$
\frac{1}{\nu(D)} \int_{D} \mathcal{H}_{\partial D}^{m}(x) d \nu(x)>-1 \quad \text { for every } \nu \text {-measurable set } D \text { such that } 0<\nu(D)<\nu(X) \text {. }
$$

Proof. Obviously, (1) implies (2), and this yields (3) since $P_{m}(D)=0$ implies, by Lemma 2.23, that $\Delta_{m} \chi_{D}=0$. Also, by Lemma 2.23, (3) implies (2). Let us now see that (2) implies (1): Suppose that $\Delta_{m}$ is not ergodic, then the space is not $m$-connected and, consequently, there exists $D \subset X$ with $\nu(D)>0$ such that $0<\nu\left(N_{D}^{m}\right)<1$; but, from Proposition 2.5,

$$
\Delta_{m} \chi_{N_{D}^{m}}(x)=m_{x}\left(N_{D}^{m}\right)-\chi_{N_{D}^{m}}(x)=0,
$$

and this implies that $\chi_{N_{D}^{m}}$ should be constant, which is a contradiction with $0<\nu\left(N_{D}^{m}\right)<\nu(X)$. The equivalence with (4) is evident by the second equivalence in Lemma 2.23.

\section{Functional Inequalities}

Let $[X, d, m]$ be a metric random walk space with invariant and reversible measure $\nu$ such that $\nu(X)<$ $+\infty$. In this section we will further assume that $\nu(X)=1$, i.e. that $\nu$ is a probability measure. Note that we may always work with $\frac{1}{\nu(X)} \nu$.

3.1. Spectral Gap and Poincaré Inequality. We denote the mean value of $f \in L^{1}(X, \nu)$ (or the expected value of $f$ ) with respect to $\nu$ by

$$
\nu(f):=\mathbb{E}_{\nu}(f)=\int_{X} f(x) d \nu(x) .
$$

Moreover, given $f \in L^{2}(X, \nu)$, we denote its variance with respect to $\nu$ by

$$
\operatorname{Var}_{\nu}(f):=\int_{X}(f(x)-\nu(f))^{2} d \nu(x)=\frac{1}{2} \int_{X \times X}(f(x)-f(y))^{2} d \nu(y) d \nu(x) .
$$

Definition 3.1. The spectral gap of $-\Delta_{m}$ is defined as

$$
\begin{aligned}
& \operatorname{gap}\left(-\Delta_{m}\right):=\inf \left\{\frac{\mathcal{H}_{m}(f)}{\operatorname{Var}_{\nu}(f)}: f \in D\left(\mathcal{H}_{m}\right), \operatorname{Var}_{\nu}(f) \neq 0\right\} \\
& =\inf \left\{\frac{\mathcal{H}_{m}(f)}{\|f\|_{2}^{2}}: f \in D\left(\mathcal{H}_{m}\right),\|f\|_{2} \neq 0, \int_{X} f d \nu=0\right\} .
\end{aligned}
$$

Observe that, since $\nu(X)<+\infty$, we have

$$
D\left(\mathcal{H}_{m}\right)=L^{2}(X, \nu) .
$$


Definition 3.2. We say that $[X, d, m, \nu]$ satisfies a Poincaré inequality if there exists $\lambda>0$ such that $\lambda \operatorname{Var}_{\nu}(f) \leq \mathcal{H}_{m}(f)$ for all $f \in L^{2}(X, \nu)$,

or, equivalently,

$$
\lambda\|f\|_{L^{2}(X, \nu)}^{2} \leq \mathcal{H}_{m}(f) \text { for all } f \in L^{2}(X, \nu) \text { with } \nu(f)=0 .
$$

Note that, if $\operatorname{gap}\left(-\Delta_{m}\right)>0$, then $[X, d, m, \nu]$ satisfies a Poincaré inequality with $\lambda=\operatorname{gap}\left(-\Delta_{m}\right)$ :

$$
\operatorname{gap}\left(-\Delta_{m}\right) \operatorname{Var}_{\nu}(f) \leq \mathcal{H}_{m}(f) \text { for all } f \in L^{2}(X, \nu),
$$

being the spectral gap the best constant in the Poincaré inequality.

With such an inequality at hand and with a similar proof to the one done in the continuous setting (see, for instance, [6]), we have that if $\operatorname{gap}\left(-\Delta_{m}\right)>0$ then $e^{t \Delta_{m}} u_{0}$ converges to $\nu\left(u_{0}\right)$ with exponential rate $\operatorname{gap}\left(-\Delta_{m}\right)$.

Theorem 3.3. The following statements are equivalent:

(i) There exists $\lambda>0$ such that

$$
\lambda \operatorname{Var}_{\nu}(f) \leq \mathcal{H}_{m}(f) \quad \text { for all } f \in L^{2}(X, \nu) .
$$

(ii) For every $f \in L^{2}(X, \nu)$

$$
\left\|e^{t \Delta_{m}} f-\nu(f)\right\|_{L^{2}(X, \nu)} \leq e^{-\lambda t}\|f-\nu(f)\|_{L^{2}(X, \nu)} \quad \text { for all } t \geq 0 ;
$$

or, equivalently, for every $f \in L^{2}(X, \nu)$ with $\nu(f)=0$,

$$
\left\|e^{t \Delta_{m}} f\right\|_{L^{2}(X, \nu)} \leq e^{-\lambda t}\|f\|_{L^{2}(X, \nu)} \text { for all } t \geq 0 .
$$

Remark 3.4. Let $\|\mu-\nu\|_{T V}$ be the total variation distance:

$$
\|\mu-\nu\|_{T V}:=\sup \{|\mu(A)-\nu(A)|: A \subset X \text { Borel }\} .
$$

Then, for $f \in L^{2}(X, \nu)$ and $\mu_{t}=e^{t \Delta_{m}} f \nu$, we have

$$
\left\|\mu_{t}-\nu\right\|_{T V} \leq\|f-1\|_{L^{2}(X, \nu)} e^{-\operatorname{gap}\left(-\Delta_{m}\right) t} .
$$

Indeed, by Theorem 3.3, for any Borel set $A \subset X$,

$$
\left|\int_{A} e^{t \Delta_{m}} f d \nu-\nu(A)\right| \leq \int_{A}\left|e^{t \Delta_{m}} f-1\right| d \nu \leq\left(\int_{X}\left|e^{t \Delta_{m}} f-1\right|^{2} d \nu\right)^{\frac{1}{2}} \leq\|f-1\|_{L^{2}(X, \nu)} e^{-\operatorname{gap}\left(-\Delta_{m}\right) t} .
$$

Hence, it is of interest to elucidate when the spectral gap of $-\Delta_{m}$ is positive. In this section we will deal with such a question.

Let $H(X, \nu)$ be the subspace of $L^{2}(X, \nu)$ consisting of the functions which are orthonormal to the constants, i.e.,

$$
H(X, \nu)=\left\{f \in L^{2}(X, \nu): \nu(f)=0\right\} .
$$

Since the operator $-\Delta_{m}: H(X, \nu) \rightarrow H(X, \nu)$ is self-adjoint and non-negative and $\left\|\Delta_{m}\right\| \leq 2$ (see Theorem 2.1), by [16, Proposition 6.9] we have that the spectrum $\sigma\left(-\Delta_{m}\right)$ of $-\Delta_{m}$ in $H(X, \nu)$ satisfies

$$
\sigma\left(-\Delta_{m}\right) \subset[\alpha, \beta] \subset[0,2],
$$


where

$$
\alpha:=\inf \left\{\left\langle-\Delta_{m} u, u\right\rangle: u \in H(X, \nu),\|u\|_{2}=1\right\} \in \sigma\left(-\Delta_{m}\right),
$$

and

$$
\beta:=\sup \left\{\left\langle-\Delta_{m} u, u\right\rangle: u \in H(X, \nu),\|u\|_{2}=1\right\} \in \sigma\left(-\Delta_{m}\right) .
$$

If $f \in L^{2}(X, \nu)$ and $\operatorname{Var}_{\nu}(f) \neq 0$, then $u:=f-\nu(f) \neq 0$ belongs to $H(X, \nu)$, so

$$
\alpha \leq \mathcal{H}_{m}\left(\frac{u}{\|u\|_{2}}\right)=\frac{\mathcal{H}_{m}(u)}{\|u\|_{2}^{2}}=\frac{\mathcal{H}_{m}(f)}{\operatorname{Var}_{\nu}(f)},
$$

and, consequently,

$$
\operatorname{gap}\left(-\Delta_{m}\right)=\alpha=\inf \left\{\left\langle-\Delta_{m} u, u\right\rangle: u \in H(X, \nu),\|u\|_{2}=1\right\}
$$

Therefore,

$$
\operatorname{gap}\left(-\Delta_{m}\right)>0 \Longleftrightarrow 0 \notin \sigma\left(-\Delta_{m}\right)
$$

If we assume that $-\Delta_{m}$ is the sum of an invertible and a compact operator in $H(X, \nu)$ (this is true, for example, if the averaging operator $M_{m}$ is compact in $H(X, \nu)$ ), then, if $0 \in \sigma\left(-\Delta_{m}\right)$, by Fredholm's alternative Theorem, we have that there exists $u \in H(X, \nu), u \neq 0$, such that $-\Delta_{m} u=\left(I-M_{m}\right) u=0$. Then, if $[X, d, m]$ is $m$-connected, by Theorem $2.21, \Delta_{m}$ is ergodic so $u$ is constant, thus $u=0$ in $H(X, \nu)$, and we get a contradiction. Consequently, we have the following result.

Proposition 3.5. Let $[X, d, m]$ be an m-connected metric random walk space with invariant-reversible probability measure $\nu$. If $-\Delta_{m}$ is the sum of an invertible operator and a compact operator in $H(X, \nu)$, then $\operatorname{gap}\left(-\Delta_{m}\right)>0$.

Example 3.6. (i) If $G=(V(G), E(G))$ is a finite weighted connected graph, then obviously $M_{m^{G}}$ is compact and, consequently, $\operatorname{gap}\left(-\Delta_{m}^{G}\right)>0$. In this situation, it is well known that, for $\sharp(V(G))=N$, the spectrum of $-\Delta_{m^{G}}$ is $0<\lambda_{1} \leq \lambda_{2} \leq \ldots \leq \lambda_{N-1}$ and $0<\lambda_{1}=\operatorname{gap}\left(-\Delta_{m}\right)$.

(ii) Another example in which $-\Delta_{m}$ is the sum of an invertible and a compact operator is $\left[\Omega, d, m^{J, \Omega}\right]$ with $\Omega$ a bounded domain and the kernel $J$ satisfying: $J \in C\left(\mathbb{R}^{N}, \mathbb{R}\right)$ is nonnegative, radially symmetric with $J(0)>0$ and $\int_{\mathbb{R}^{N}} J(x) d x=1$. Indeed,

$$
-\Delta_{m^{J, \Omega}} f(x)=\int_{\Omega} J(x-y) d y f(x)-\int_{\Omega} J(x-y) f(y) d y,
$$

where $\int_{\Omega} J(x-y) d y f(x)$ defines an invertible operator and $\int_{\Omega} J(x-y) f(y) d y$ defines a compact operator. Hence, in this case we have (see also [4]):

$$
\operatorname{gap}\left(-\Delta_{m^{J, \Omega}}\right)=\inf \left\{\frac{\frac{1}{2} \int_{\Omega \times \Omega} J(x-y)(u(y)-u(x))^{2} d x d y}{\int_{\Omega} u(x)^{2} d x}: u \in L^{2}(\Omega),\|u\|_{L^{2}(X, \nu)}>0, \int_{\Omega} u=0\right\}>0 .
$$

Let us point out that the condition $J(0)>0$ is necessary, see [4, Remark 6.20].

As a consequence of a result by Miclo [41], we have that $\operatorname{gap}\left(-\Delta_{m}\right)>0$ if $\Delta_{m}$ is ergodic and $M_{m}$ is hyperbounded, that is, if there exists $p>2$ such that $M_{m}$ is bounded from $L^{2}(X, \nu)$ to $L^{p}(X, \nu)$. If we 
have that $m_{x} \ll \nu$, i.e., $m_{x}=f_{x} \nu$ with $f_{x} \in L^{1}(X, \nu)$, and we assume that

$$
\int_{X}\left\|f_{x}\right\|_{L^{2}(X, \nu)}^{p} d \nu(x)=K<\infty
$$

then, for $u \in L^{2}(X, \nu)$, by the Cauchy-Schwarz inequality, we have that

$$
\begin{aligned}
\left\|M_{m} u\right\|_{p}^{p}=\int_{X}\left|M_{m} u(x)\right|^{p} d \nu(x) & =\int_{X}\left|\int_{X} u(y) d m_{x}(y)\right|^{p} d \nu(x)=\int_{X}\left|\int_{X} u(y) f_{x}(y) d \nu(y)\right|^{p} d \nu(x) \\
\leq & \|u\|_{L^{2}(X, \nu)}^{p} \int_{X}\left\|f_{x}\right\|_{L^{2}(X, \nu)}^{p} d \nu(x),
\end{aligned}
$$

hence

$$
\left\|M_{m} u\right\|_{p} \leq K^{\frac{1}{p}}\|u\|_{L^{2}(X, \nu)}
$$

Therefore, $M_{m}$ is hyperbounded and, consequently, we have the following result about the spectral gap.

Proposition 3.7. If $\Delta_{m}$ is ergodic and (3.3) holds, then $\operatorname{gap}\left(-\Delta_{m}\right)>0$.

In the next example we see that there exist metric random walk spaces for which the Poincaré inequality does not hold.

Example 3.8. Let $V(G)=\left\{x_{3}, x_{4}, x_{5} \ldots, x_{n} \ldots\right\}$ be a weighted linear graph with

$$
w_{x_{3 n}, x_{3 n+1}}=\frac{1}{n^{3}}, w_{x_{3 n+1}, x_{3 n+2}}=\frac{1}{n^{2}}, w_{x_{3 n+2}, x_{3 n+3}}=\frac{1}{n^{3}},
$$

for $n \geq 1$, and let

$$
f_{n}(x)= \begin{cases}n & \text { if } x=x_{3 n+1}, x_{3 n+2} \\ 0 & \text { else. }\end{cases}
$$

Note that $\nu(X)<+\infty$ (we avoid its normalization for simplicity). Now,

$$
\begin{gathered}
2 \mathcal{H}_{m}\left(f_{n}\right)=\int_{X} \int_{X}\left(f_{n}(x)-f_{n}(y)\right)^{2} d m_{x}(y) d \nu(x) \\
=d_{x_{3 n}} \int_{X}\left(f_{n}\left(x_{3 n}\right)-f_{n}(y)\right)^{2} d m_{x_{3 n}}(y)+d_{x_{3 n+1}} \int_{X}\left(f_{n}\left(x_{3 n+1}\right)-f_{n}(y)\right)^{2} d m_{x_{3 n+1}}(y) \\
+d_{x_{3 n+2}} \int_{X}\left(f_{n}\left(x_{3 n+2}\right)-f_{n}(y)\right)^{2} d m_{x_{3 n+2}}(y)+d_{x_{3 n+3}} \int_{X}\left(f_{n}\left(x_{3 n+3}\right)-f_{n}(y)\right)^{2} d m_{x_{3 n+3}}(y) \\
=d_{x_{3 n}} n^{2} \frac{\frac{1}{n^{3}}}{d_{x_{3 n}}}+d_{x_{3 n+1}} n^{2} \frac{\frac{1}{n^{3}}}{d_{x_{3 n+1}}}+d_{x_{3 n+2}} n^{2} \frac{\frac{1}{n^{3}}}{d_{x_{3 n+2}}}+d_{x_{3 n+3}} n^{2} \frac{\frac{1}{n^{3}}}{d_{x_{3 n+3}}}=\frac{4}{n} .
\end{gathered}
$$

However, we have

$$
\int_{X} f_{n}(x) d \nu(x)=n\left(d_{x_{3 n+1}}+d_{x_{3 n+2}}\right)=2 n\left(\frac{1}{n^{2}}+\frac{1}{n^{3}}\right)=\frac{2}{n}\left(1+\frac{1}{n}\right),
$$

thus

$$
\nu\left(f_{n}\right)=\frac{\frac{2}{n}\left(1+\frac{1}{n}\right)}{\nu(X)}=\widetilde{O}\left(\frac{1}{n}\right),
$$

where we use the notation

$$
\varphi(n)=\widetilde{O}(\psi(n)) \Longleftrightarrow \exists \lim _{n \rightarrow \infty} \frac{\varphi(n)}{\psi(n)}=C \neq 0 .
$$


Therefore,

$$
\left(f_{n}(x)-\nu\left(f_{n}\right)\right)^{2}= \begin{cases}\widetilde{O}\left(n^{2}\right) & \text { if } x=x_{3 n+1}, x_{3 n+2} \\ \widetilde{O}\left(\frac{1}{n^{2}}\right) & \text { otherwise }\end{cases}
$$

Finally,

$$
\begin{gathered}
\operatorname{Var}_{\nu}\left(f_{n}\right)=\int_{X}\left(f_{n}(x)-\nu\left(f_{n}\right)\right)^{2} d \nu(x)=\widetilde{O}\left(\frac{1}{n^{2}}\right) \sum_{x \neq x_{3 n+1}, x_{3 n+2}} d_{x}+\widetilde{O}\left(n^{2}\right)\left(d_{x_{3 n+1}}+d_{x_{3 n+2}}\right) \\
=\widetilde{O}\left(\frac{1}{n^{2}}\right)+2 \widetilde{O}\left(n^{2}\right)\left(\frac{1}{n^{2}}+\frac{1}{n^{3}}\right)=\widetilde{O}(1) .
\end{gathered}
$$

Consequently, $\left[V(G), d_{G},\left(m_{x}\right), \nu\right]$ does not satisfy a Poincaré inequality for any $\lambda>0$.

In general, since $\mathcal{H}_{m}(f)=-\int_{X} f(x) \Delta_{m} f(x) d \nu(x)$, if $\Delta_{m} f=0$ then $\mathcal{H}_{m}(f)=0$ and, therefore, if $[X, d, m, \nu]$ satisfies a Poincaré inequality, we have that $f$ is constant:

$$
f(x)=\int_{X} f(x) d \nu(x) \quad \nu \text { - a.e. }
$$

Consequently, we get the following result.

Proposition 3.9. If $[X, d, m, \nu]$ satisfies a Poincaré inequality we have that $\Delta_{m}$ is ergodic.

Example 3.8 shows that the reverse implication does not hold in general.

3.2. Isoperimetric Inequality. Recall that, for a $\nu$-measurable set $D \subset X$,

$$
P_{m}(D)=\int_{E} \int_{X \backslash E} d m_{x}(y) d \nu(x)=T V_{m}\left(\chi_{E}\right) .
$$

The Poincaré inequality, if given only for characteristic functions, implies that there exists $\lambda>0$ such that

$$
\lambda \nu(D)(1-\nu(D)) \leq P_{m}(D) \text { for every } \nu-\text { measurable set } D,
$$

(observe that this also implies the ergodicity of $\Delta_{m}$, as we have seen in Theorem 2.24). Hence, since

$$
\min \{x, 1-x\} \leq 2 x(1-x) \leq 2 \min \{x, 1-x\} \text { for } 0 \leq x \leq 1,
$$

inequality (3.4) implies the following isoperimetric inequality (see [1, Theorem 3.46]):

$$
\min \{\nu(D), 1-\nu(D)\} \leq \frac{2}{\lambda} P_{m}(D) \quad \text { for every } \nu \text {-measurable set } D
$$

and, conversely, the isoperimetric inequality (3.5) implies

$$
\frac{\lambda}{2} \nu(D)(1-\nu(D)) \leq P_{m}(D) \text { for every } \nu \text {-measurable set } D .
$$

Definition 3.10. If there exists $\lambda>0$ satisfying (3.5), we say that $[X, d, m, \nu]$ satisfies an isoperimetric inequality. 
3.3. Cheeger Inequality. In a weighted graph $G=(V(G), E(G))$ the Cheeger constant is defined as

$$
h_{G}:=\inf _{D \subset V(G)} \frac{|\partial D|}{\min \left\{\nu_{G}(D), \nu_{G}(V(G) \backslash D)\right\}},
$$

where

$$
|\partial D|:=\sum_{x \in D, y \in V \backslash D} w_{x y} .
$$

In [19] (see also [10]), the following relation between the Cheeger constant and the first positive eigenvalue $\lambda_{1}(G)$ of the graph Laplacian $\Delta_{m^{G}}$ is proved:

$$
\frac{h_{G}^{2}}{2} \leq \lambda_{1}(G) \leq 2 h_{G} .
$$

The previous inequality appeared in [17], and can be traced back to the paper by Polya and Szego [47].

Let $[X, d, m]$ be a metric random walk space with invariant and reversible probability measure $\nu$. We define its Cheeger constant as

$$
h_{m}(X):=\inf \left\{\frac{P_{m}(D)}{\min \{\nu(D), \nu(X \backslash D)\}}: D \subset X, 0<\nu(D)<1\right\},
$$

or, equivalently,

$$
h_{m}(X)=\inf \left\{\frac{P_{m}(D)}{\nu(D)}: D \subset X, 0<\nu(D) \leq \frac{1}{2}\right\} .
$$

Having in mind (1.4), we have that this definition is consistent with the definition on graphs. Note that, if $h_{m}(X)>0$, then $h_{m}(X)$ is the best constant in the isoperimetric inequality (3.5).

We will now give a variational characterization of the Cheeger constant which generalizes the one obtained in [52] for the particular case of finite graphs. Recall that, given a function $u: X \rightarrow \mathbb{R}, \mu \in \mathbb{R}$ is a median of $u$ with respect to a measure $\nu$ if

$$
\nu(\{x \in X: u(x)<\mu\}) \leq \frac{1}{2} \nu(X), \quad \nu(\{x \in X: u(x)>\mu\}) \leq \frac{1}{2} \nu(X) .
$$

We denote by $\operatorname{med}_{\nu}(u)$ the set of all medians of $u$. It is easy to see that

$$
\mu \in \operatorname{med}_{\nu}(u) \Longleftrightarrow-\nu(\{u=\mu\}) \leq \nu(\{x \in X: u(x)>\mu\})-\nu(\{x \in X: u(x)<\mu\}) \leq \nu(\{u=\mu\}),
$$

from where it follows that

$$
0 \in \operatorname{med}_{\nu}(u) \Longleftrightarrow \exists \xi \in \operatorname{sign}(u) \text { such that } \int_{X} \xi(x) d \nu(x)=0
$$

where

$$
\operatorname{sign}(u)(x):= \begin{cases}1 & \text { if } u(x)>0 \\ -1 & \text { if } u(x)<0 \\ {[-1,1]} & \text { if } u=0\end{cases}
$$

Let

$$
\lambda_{1}^{m}(X):=\inf \left\{T V_{m}(u):\|u\|_{1}=1,0 \in \operatorname{med}_{\nu}(u)\right\} .
$$

Theorem 3.11. If $[X, d, m]$ is a metric random walk space with invariant and reversible probability measure $\nu$, then

$$
h_{m}(X)=\lambda_{1}^{m}(X)
$$


Proof. If $D \subset X, 0<\nu(D) \leq \frac{1}{2}$, then $0 \in \operatorname{med}_{\nu}\left(\chi_{D}\right)$. Thus,

$$
\lambda_{1}^{m}(X) \leq T V_{m}\left(\frac{1}{\nu(D)} \chi_{D}\right)=\frac{1}{\nu(D)} P_{m}(D)
$$

and, therefore,

$$
\lambda_{1}^{m}(X) \leq h_{m}(X) .
$$

Now, for the other inequality, let $u \in L^{1}(X, \nu)$ such that $\|u\|_{1}=1$ and $0 \in \operatorname{med}_{\nu}(u)$. $\operatorname{Since}_{0} \in \operatorname{med}_{\nu}(u)$, by the Coarea formula (Theorem 1.4), and having in mind that the set $\{t \in \mathbb{R}: \nu(\{u=t\})>0\}$ is countable, we have

$$
\begin{gathered}
T V_{m}(u)=\int_{-\infty}^{+\infty} P_{m}\left(E_{t}(u)\right) d t=\int_{0}^{+\infty} P_{m}\left(E_{t}(u)\right) d t+\int_{-\infty}^{0} P_{m}\left(X \backslash E_{t}(u)\right) d t \\
\geq h_{m}(X) \int_{0}^{+\infty} \nu\left(E_{t}(u)\right) d t+h_{m}(X) \int_{-\infty}^{0} \nu\left(X \backslash E_{t}(u)\right) d t \\
=h_{m}(X)\left(\int_{X} u^{+}(x) d \nu(x)+\int_{X} u^{-}(x) d \nu(x)\right)=h_{m}(X)\|u\|_{1}=h_{m}(X) .
\end{gathered}
$$

Therefore, taking the infimum in $u$, we get $\lambda_{1}^{m}(X) \geq h_{m}(X)$.

Following [19] and using Theorem 3.11, in the next result we see that the Cheeger inequality (3.6) also holds in our context.

Theorem 3.12. Let $[X, d, m]$ be a metric random walk space with invariant and reversible probability measure $\nu$. The following Cheeger inequality holds

$$
\frac{h_{m}^{2}}{2} \leq \operatorname{gap}\left(-\Delta_{m}\right) \leq 2 h_{m} .
$$

Proof. Let $\left(f_{n}\right) \subset D\left(\mathcal{H}_{m}\right)$, with $\nu\left(f_{n}\right)=0$, such that

$$
\lim _{n \rightarrow \infty} \frac{\mathcal{H}_{m}\left(f_{n}\right)}{\left\|f_{n}\right\|_{2}^{2}}=\operatorname{gap}\left(-\Delta_{m}\right)
$$

If we take $\mu_{n} \in \operatorname{med}_{\nu}\left(f_{n}\right)$, we have

$$
\begin{aligned}
& 2 \mathcal{H}_{m}\left(f_{n}\right)=\int_{X} \int_{X}\left(f_{n}(y)-\mu_{n}-\left(f_{n}(x)-\mu_{n}\right)\right)^{2} d m_{x}(y) d \nu(x) \\
& =\int_{X} \int_{X}\left[\left(f_{n}(y)-\mu_{n}\right)^{+}-\left(f_{n}(x)-\mu_{n}\right)^{+}-\left(\left(f_{n}(y)-\mu_{n}\right)^{-}-\left(f_{n}(x)-\mu_{n}\right)^{-}\right)\right]^{2} d m_{x}(y) d \nu(x) \\
& =\int_{X} \int_{X}\left(\left(f_{n}(y)-\mu_{n}\right)^{+}-\left(f_{n}(x)-\mu_{n}\right)^{+}\right)^{2} d m_{x}(y) d \nu(x) \\
& \quad+\int_{X} \int_{X}\left(\left(f_{n}(y)-\mu_{n}\right)^{-}-\left(f_{n}(x)-\mu_{n}\right)^{-}\right)^{2} d m_{x}(y) d \nu(x) \\
& \quad-2 \int_{X} \int_{X}\left(\left(f_{n}(y)-\mu_{n}\right)^{+}-\left(f_{n}(x)-\mu_{n}\right)^{+}\right)\left(\left(f_{n}(y)-\mu_{n}\right)^{-}-\left(f_{n}(x)-\mu_{n}\right)^{-}\right) d m_{x}(y) d \nu(x) .
\end{aligned}
$$


Now, an easy calculation gives

$$
-\int_{X} \int_{X}\left(\left(f_{n}(y)-\mu_{n}\right)^{+}-\left(f_{n}(x)-\mu_{n}\right)^{+}\right)\left(\left(f_{n}(y)-\mu_{n}\right)^{-}-\left(f_{n}(x)-\mu_{n}\right)^{-}\right) d m_{x}(y) d \nu(x) \geq 0 .
$$

On the other hand, since $\nu\left(f_{n}\right)=0$, we have

$$
\int_{X} f_{n}^{2}(x) d \nu(x) \leq \int_{X}\left(f_{n}(x)-\mu_{n}\right)^{2} d \nu(x) .
$$

Therefore,

$$
\begin{aligned}
\frac{2 \mathcal{H}_{m}\left(f_{n}\right)}{\left\|f_{n}\right\|_{2}^{2}} \geq & \frac{\int_{X} \int_{X}\left(\left(f_{n}(y)-\mu_{n}\right)^{+}-\left(f_{n}(x)-\mu_{n}\right)^{+}\right)^{2} d m_{x}(y) d \nu(x)}{\int_{X}\left[\left(f_{n}(x)-\mu_{n}\right)^{+}\right]^{2} d \nu(x)+\int_{X}\left[\left(f_{n}(x)-\mu_{n}\right)^{-}\right]^{2} d \nu(x)}+ \\
& +\frac{\int_{X} \int_{X}\left(\left(f_{n}(y)-\mu_{n}\right)^{-}-\left(f_{n}(x)-\mu_{n}\right)^{-}\right)^{2} d m_{x}(y) d \nu(x)}{\int_{X}\left[\left(f_{n}(x)-\mu_{n}\right)^{+}\right]^{2} d \nu(x)+\int_{X}\left[\left(f_{n}(x)-\mu_{n}\right)^{-}\right]^{2} d \nu(x)} .
\end{aligned}
$$

Having in mind that

$$
\frac{a+b}{c+d} \geq \min \left\{\frac{a}{c}, \frac{b}{d}\right\} \quad \text { for every } a, b, c, d \in \mathbb{R}^{+}
$$

and

$$
\int_{X}\left[\left(f_{n}(x)-\mu_{n}\right)^{+}\right]^{2} d \nu(x)+\int_{X}\left[\left(f_{n}(x)-\mu_{n}\right)^{-}\right]^{2} d \nu(x)>0,
$$

we can assume, without loss of generality, that

$$
\int_{X}\left[\left(f_{n}(x)-\mu_{n}\right)^{+}\right]^{2} d \nu(x)>0,
$$

and that

$$
\frac{2 \mathcal{H}_{m}\left(f_{n}\right)}{\left\|f_{n}\right\|_{2}^{2}} \geq \frac{\int_{X} \int_{X}\left(\left(f_{n}(y)-\mu_{n}\right)^{+}-\left(f_{n}(x)-\mu_{n}\right)^{+}\right)^{2} d m_{x}(y) d \nu(x)}{\int_{X}\left[\left(f_{n}(x)-\mu_{n}\right)^{+}\right]^{2} d \nu(x)} .
$$

By the Cauchy-Schwartz inequality, we have

$$
\begin{aligned}
& \int_{X} \int_{X}\left|\left[\left(f_{n}(y)-\mu_{n}\right)^{+}\right]^{2}-\left[\left(f_{n}(x)-\mu_{n}\right)^{+}\right]^{2}\right| d m_{x}(y) d \nu(x) \\
& =\int_{X} \int_{X}\left|\left(f_{n}(y)-\mu_{n}\right)^{+}-\left(f_{n}(x)-\mu_{n}\right)^{+}\right|\left|\left(f_{n}(y)-\mu_{n}\right)^{+}+\left(f_{n}(x)-\mu_{n}\right)^{+}\right| d m_{x}(y) d \nu(x) \\
& \leq\left(\int_{X} \int_{X}\left(\left(f_{n}(y)-\mu_{n}\right)^{+}-\left(f_{n}(x)-\mu_{n}\right)^{+}\right)^{2} d m_{x}(y) d \nu(x)\right)^{\frac{1}{2}} \times \\
& \quad \times\left(\int_{X} \int_{X}\left(\left(f_{n}(y)-\mu_{n}\right)^{+}+\left(f_{n}(x)-\mu_{n}\right)^{+}\right)^{2} d m_{x}(y) d \nu(x)\right)^{\frac{1}{2}} .
\end{aligned}
$$


Now, by the invariance of $\nu$,

$$
\int_{X} \int_{X}\left(\left(f_{n}(y)-\mu_{n}\right)^{+}+\left(f_{n}(x)-\mu_{n}\right)^{+}\right)^{2} d m_{x}(y) d \nu(x) \leq 4 \int_{X}\left[\left(f_{n}(x)-\mu_{n}\right)^{+}\right]^{2} d \nu(x) .
$$

Thus

$$
\frac{2 \mathcal{H}_{m}\left(f_{n}\right)}{\left\|f_{n}\right\|_{2}^{2}} \geq\left(\frac{\frac{1}{2} \int_{X} \int_{X}\left|\left[\left(f_{n}(y)-\mu_{n}\right)^{+}\right]^{2}-\left[\left(f_{n}(x)-\mu_{n}\right)^{+}\right]^{2}\right| d m_{x}(y) d \nu(x)}{\int_{X}\left[\left(f_{n}(x)-\mu_{n}\right)^{+}\right]^{2} d \nu(x)}\right)^{2} .
$$

Then, since $0 \in \operatorname{med}_{\nu}\left(\left[\left(f_{n}-\mu_{n}\right)^{+}\right]^{2}\right)$, by Theorem 3.11, we get

$$
\frac{2 \mathcal{H}_{m}\left(f_{n}\right)}{\left\|f_{n}\right\|_{2}^{2}} \geq h_{m}(X)^{2}
$$

and, consequently, taking limits as $n \rightarrow \infty$, we obtain

$$
\frac{h_{m}^{2}}{2} \leq \operatorname{gap}\left(-\Delta_{m}\right)
$$

To prove the other inequality we can assume that $\operatorname{gap}\left(-\Delta_{m}\right)>0$. Now, by (3.5), we have

$$
\min \{\nu(D), 1-\nu(D)\} \leq \frac{2}{\operatorname{gap}\left(-\Delta_{m}\right)} P_{m}(D) \quad \text { for all } D \subset X, 0<\nu(D)<1,
$$

from where it follows that $\operatorname{gap}\left(-\Delta_{m}\right) \leq 2 h_{m}(X)$.

Let $A \subset X$ with $\nu(A)=\frac{1}{2}$ and $u=\chi_{A}-\chi_{X \backslash A}$. It is easy to see that $T V_{m}(u)=2 P_{m}(A)$ and $\mathcal{H}_{m}(u)=4 P_{m}(A)$. Hence, since $\|u\|_{1}=\|u\|_{2}=1, \nu(u)=0$ and $0 \in \operatorname{med}_{\nu}(u)$, we obtain the following result as a consequence of Theorem 3.11.

Corollary 3.13. Let $[X, d, m]$ be a metric random walk space with invariant and reversible probability measure $\nu$. Let $A \subset X$ with $\nu(A)=\frac{1}{2}$ and $u=\chi_{A}-\chi_{X \backslash A}$. Then,

1. $h_{m}(X)=\frac{P_{m}(A)}{\nu(A)} \Longleftrightarrow u=\chi_{A}-\chi_{X \backslash A}$ is a minimizer of (3.7).

2. $u$ is a minimizer of (3.7) and $\operatorname{gap}\left(-\Delta_{m}\right)=2 h_{m}(X) \Longleftrightarrow u$ is a minimizer of (3.1).

Bringing together all the above results we have:

Theorem 3.14. Let $[X, d, m]$ be a metric random walk space with invariant and reversible probability measure $\nu$. The following statements are equivalent:

(1) $[X, d, m, \nu]$ satisfies a Poincaré inequality,

(2) $\operatorname{gap}\left(-\Delta_{m}\right)>0$,

(3) $[X, d, m, \nu]$ satisfies an isoperimetric inequality,

(4) $h_{m}(X)>0$.

Example 3.15. It is well known, (see for instance [19]) that for finite graphs $G, h_{m}(G)>0$ if, and only if, $G$ is connected. This result is not true for infinite graphs. In fact, the graph of the Example 3.8 is connected and its Cheeger constant is zero since its spectral gap is zero. 
3.4. Spectral Gap and Curvature. Since $\mathcal{E}_{m}$ admits a Carré du champ $\Gamma$ (see [6]) defined by

$$
\Gamma(f, g)(x):=\frac{1}{2}\left(\Delta_{m}(f g)(x)-f(x) \Delta_{m} g(x)-g(x) \Delta_{m} f(x)\right) \text { for all } x \in X \text { and } f, g \in L^{2}(X, \nu),
$$

we can study the Bakry-Émery curvature-dimension condition in this context. We will study its relation with the spectral gap.

According to Bakry and Émery [7], we define the Ricci curvature operator $\Gamma_{2}$ by iterating $\Gamma$ :

$$
\Gamma_{2}(f, g):=\frac{1}{2}\left(\Delta_{m} \Gamma(f, g)-\Gamma\left(f, \Delta_{m} g\right)-\Gamma\left(\Delta_{m} f, g\right)\right),
$$

which is well defined for $f, g \in L^{2}(X, \nu)$. We will write, for $f \in L^{2}(X, \nu)$,

$$
\Gamma(f):=\Gamma(f, f)=\frac{1}{2} \Delta_{m}\left(f^{2}\right)-f \Delta_{m} f
$$

and

$$
\Gamma_{2}(f):=\Gamma_{2}(f, f)=\frac{1}{2} \Delta_{m} \Gamma(f)-\Gamma\left(f, \Delta_{m} f\right) .
$$

It is easy to see that

$$
\Gamma(f, g)(x)=\frac{1}{2} \int_{X} \nabla f(x, y) \nabla g(x, y) d m_{x}(y) \quad \text { and } \quad \Gamma(f)(x)=\frac{1}{2} \int_{X}|\nabla f(x, y)|^{2} d m_{x}(y) .
$$

Consequently,

$$
\int_{X} \Gamma(f, g)(x) d \nu(x)=\mathcal{E}_{m}(f, g) \quad \text { and } \quad \int_{X} \Gamma(f)(x) d \nu(x)=\mathcal{H}_{m}(f) .
$$

Furthermore, by (2.1) and (3.8), we get

$$
\int_{X} \Gamma_{2}(f) d \nu=\frac{1}{2} \int_{X}\left(\Delta_{m} \Gamma(f)-2 \Gamma\left(f, \Delta_{m} f\right)\right) d \nu=-\int_{X} \Gamma\left(f, \Delta_{m} f\right) d \nu=-\mathcal{E}_{m}\left(f, \Delta_{m} f\right),
$$

thus

$$
\int_{X} \Gamma_{2}(f) d \nu=\int_{X}\left(\Delta_{m} f\right)^{2} d \nu
$$

Definition 3.16. The operator $\Delta_{m}$ satisfies the Bakry-Émery curvature-dimension condition $B E(K, n)$ for $n \in(1,+\infty)$ and $K \in \mathbb{R}$ if

$$
\Gamma_{2}(f) \geq \frac{1}{n}\left(\Delta_{m} f\right)^{2}+K \Gamma(f) \quad \forall f \in L^{2}(X, \nu) .
$$

The constant $n$ is the dimension of the operator $\Delta_{m}$, and $K$ is the lower bound of the Ricci curvature of the operator $\Delta_{m}$. If there exists $K \in \mathbb{R}$ such that

$$
\Gamma_{2}(f) \geq K \Gamma(f) \quad \forall f \in L^{2}(X, \nu),
$$

then it is said that the operator $\Delta_{m}$ satisfies the Bakry-Émery curvature-dimension condition $B E(K, \infty)$.

Observe that if $\Delta_{m}$ satisfies the Bakry-Émery curvature-dimension condition $B E(K, n)$ then it also satisfies the Bakry-Émery curvature-dimension condition $B E(K, m)$ for $m>n$.

This definition is motivated by the well known fact that on a complete $n$-dimensional Riemannian manifold $(M, g)$, the Laplace-Beltrami operator $\Delta_{g}$ satisfies $B E(K, n)$ if, and only if, the Ricci curvature of the Riemannian manifold is bounded from below by $K$ (see, for example, [6, Appendix C.6]). 
The use of the Bakry-Émery curvature-dimension condition as a possible definition of a Ricci curvature bound in Markov chains was first considered in 1998 [50]. Now, this concept of Ricci curvature in the discrete setting has been frequently used since the work by Lin and Yau [33] (see [31] and the references therein).

Integrating the Bakry-Émery curvature-dimension condition $B E(K, n)$ we have

$$
\int_{X} \Gamma_{2}(f) d \nu \geq \frac{1}{n} \int_{X}\left(\Delta_{m} f\right)^{2} d \nu+K \int_{X} \Gamma(f) d \nu
$$

Now, by (3.8) and (3.9), this inequality can be rewritten as

$$
\int_{X}\left(\Delta_{m} f\right)^{2} d \nu \geq \frac{1}{n} \int_{X}\left(\Delta_{m} f\right)^{2} d \nu+K \mathcal{H}_{m}(f),
$$

or, equivalently, as

$$
K \frac{n}{n-1} \mathcal{H}_{m}(f) \leq \int_{X}\left(\Delta_{m} f\right)^{2} d \nu
$$

Similarly, integrating the Bakry-Émery curvature-dimension condition $B E(K, \infty)$ we have

$$
K \mathcal{H}_{m}(f) \leq \int_{X}\left(\Delta_{m} f\right)^{2} d \nu
$$

We call the inequalities (3.10) and (3.11) the integrated Bakry-Émery curvature-dimension conditions, and denote them by $\operatorname{IBE}(K, n)$ and $\operatorname{IBE}(K, \infty)$, respectively.

Theorem 3.17. Let $[X, d, m]$ be a metric random walk space with invariant-reversible probability measure $\nu$. Assume that $\Delta_{m}$ is ergodic. Then

$$
\operatorname{gap}\left(-\Delta_{m}\right)=\sup \left\{\lambda \geq 0: \lambda \mathcal{H}_{m}(f) \leq \int_{X}\left(-\Delta_{m} f\right)^{2} d \nu \quad \forall f \in L^{2}(X, \nu)\right\} .
$$

Proof. By (3.2) we know that $\operatorname{gap}\left(-\Delta_{m}\right)=\alpha$. Set

$$
A:=\sup \left\{\lambda \geq 0: \lambda \mathcal{H}_{m}(f) \leq \int_{X}\left(-\Delta_{m} f\right)^{2} d \nu \quad \forall f \in L^{2}(X, \nu)\right\} .
$$

Let $\left(P_{\lambda}\right)_{\lambda \geq 0}$ be the spectral projection of the self-adjoint and positive operator $-\Delta_{m}: H(X, \nu) \rightarrow$ $H(X, \nu)$. By the spectral Theorem [48, Theorem VIII. 6], we have

$$
\begin{gathered}
\mathcal{H}_{m}(f)=\left\langle-\Delta_{m} f, f\right\rangle=\int_{\alpha}^{\beta} \lambda d\left\langle P_{\lambda} f, f\right\rangle \\
\int_{X}\left(-\Delta_{m} f\right)^{2} d \nu=\left\langle-\Delta_{m} f,-\Delta_{m} f\right\rangle=\int_{\alpha}^{\beta} \lambda^{2} d\left\langle P_{\lambda} f, f\right\rangle .
\end{gathered}
$$

Hence, since $\lambda^{2} \geq \alpha \lambda$, we have that

$$
\int_{X}\left(-\Delta_{m} f\right)^{2} d \nu \geq \alpha \int_{\alpha}^{\beta} \lambda d\left\langle P_{\lambda} f, f\right\rangle=\alpha \mathcal{H}_{m}(f),
$$

and we get $\alpha \leq A$. Finally, let us see that $\alpha \geq A$. Since $\alpha \in \sigma\left(\Delta_{m}\right)$, given $\epsilon>0$, there exists $0 \neq f \in \operatorname{Range}\left(P_{\alpha+\epsilon}\right)$ and, consequently, $P_{\lambda} f=f$ for $\lambda \geq \alpha+\epsilon$. Then, having in mind the ergodicity of 
$\Delta_{m}$, we have

$$
0<\int_{X}\left(-\Delta_{m} f\right)^{2} d \nu=\int_{\alpha}^{\alpha+\epsilon} \lambda^{2} d\left\langle P_{\lambda} f, f\right\rangle \leq(\alpha+\epsilon) \int_{\alpha}^{\alpha+\epsilon} \lambda d\left\langle P_{\lambda} f, f\right\rangle=(\alpha+\epsilon) \mathcal{H}_{m}(f)<(\alpha+2 \epsilon) \mathcal{H}_{m}(f) .
$$

This implies that $\alpha+2 \epsilon$ does not belong to the set

$$
\left\{\lambda \geq 0: \lambda \mathcal{H}_{m}(f) \leq \int_{X}\left(-\Delta_{m} f\right)^{2} d \nu \quad \forall f \in L^{2}(X, \nu)\right\},
$$

thus $A<\alpha+2 \epsilon$. Therefore, since $\epsilon>0$ was arbitrary, we have

$$
A \leq \alpha .
$$

Consequently, on account of Theorem 3.17, we can rewrite the Poincaré inequality via the integrated Bakry-Émery curvature-dimension condition (see [6, Theorem 4.8.4], see also [9, Theorem 2.1]):

Theorem 3.18. Let $[X, d, m]$ be a metric random walk space with invariant-reversible probability measure $\nu$. Assume that $\Delta_{m}$ is ergodic. Then,

(1) $\Delta_{m}$ satisfies an integrated Bakry-Émery curvature-dimension condition $\operatorname{IBE}(K, n)$ with $K>0$ if, and only if, a Poincaré inequality with constant $K \frac{n}{n-1}$ is satisfied.

(2) $\Delta_{m}$ satisfies an integrated Bakry-Émery curvature-dimension condition $\operatorname{IBE}(K, \infty)$ with $K>0$ if, and only if, a Poincaré inequality with constant $K$ is satisfied.

Therefore, if $\Delta_{m}$ satisfies the Bakry-Émery curvature-dimension condition $B E(K, n)$ with $K>0$, we have

$$
\operatorname{gap}\left(-\Delta_{m}\right) \geq K \frac{n}{n-1} .
$$

In the case that $\Delta_{m}$ satisfies the Bakry-Émery curvature-dimension condition $B E(K, \infty)$ with $K>0$, we have

$$
\operatorname{gap}\left(-\Delta_{m}\right) \geq K
$$

In the next example we will see that, in general, the integrated Bakry-Émery curvature-dimension condition $I B E(K, n)$ with $K>0$ does not imply the Bakry-Émery curvature-dimension condition $B E(K, n)$ with $K>0$.

Example 3.19. Consider the weighted linear graph $G$ with vertex set $V(G)=\{a, b, c\}$ and where the only non-zero weights are $w_{a, b}=w_{b, c}=1$, and let $\Delta:=\Delta_{m^{G}}$. A simple calculation gives

$$
\begin{gathered}
\Gamma(f)(a)=\frac{1}{2}(f(b)-f(a))^{2}=\frac{1}{2}(\Delta f(a))^{2}, \\
\Gamma(f)(c)=\frac{1}{2}(f(b)-f(c))^{2}=\frac{1}{2}(\Delta f(c))^{2}, \\
\Gamma(f)(b)=\frac{1}{4}(f(b)-f(a))^{2}+\frac{1}{4}(f(b)-f(c))^{2}=\frac{1}{4}\left((\Delta f(a))^{2}+(\Delta f(c))^{2}\right)=\frac{1}{2}(\Gamma(f)(a)+\Gamma(f)(c)) .
\end{gathered}
$$

Moreover,

and

$$
\Gamma_{2}(f)(a)=\frac{1}{8}(\Delta f(c))^{2}+\frac{5}{8}(\Delta f(a))^{2}+\frac{1}{4} \Delta f(a) \Delta f(c)
$$

$$
\Gamma_{2}(f)(c)=\frac{1}{8}(\Delta f(a))^{2}+\frac{5}{8}(\Delta f(c))^{2}+\frac{1}{4} \Delta f(a) \Delta f(c) .
$$


Having in mind (3.14) and (3.15), the $B E(K, n)$ condition

$$
\Gamma_{2}(f) \geq \frac{1}{n}(\Delta f)^{2}+K \Gamma(f) \quad \forall f \in L^{2}(X, \nu)
$$

on $a$ or $c$ holds true if, and only if,

$$
\frac{1}{4} y^{2}+\frac{5}{4} x^{2}+\frac{1}{2} x y \geq \frac{2}{n} x^{2}+K x^{2} \quad \forall x, y \in \mathbb{R} .
$$

Now, since (3.16) is true for $x=0,(3.16)$ holds if, and only if,

$$
K \leq \inf _{x \neq 0, y} \frac{\frac{1}{4} y^{2}+\frac{5}{4} x^{2}+\frac{1}{2} x y-\frac{2}{n} x^{2}}{x^{2}} .
$$

Moreover, taking $y=\lambda x$, we obtain that the following inequality must be satisfied

$$
K \leq \inf _{\lambda}\left(\frac{1}{4} \lambda^{2}+\frac{5}{4}+\frac{1}{2} \lambda-\frac{2}{n}\right)=1-\frac{2}{n} .
$$

In fact, it is easy to see that (3.16) is true for any $K \leq 1-\frac{2}{n}$.

On the other hand, we have that

$$
\Gamma_{2}(f)(b)=\frac{1}{2}(\Delta f(b))^{2}+\Gamma(f)(b),
$$

and it is easy to see that

$$
\Gamma_{2}(f)(b) \geq \frac{1}{n}(\Delta f(b))^{2}+K \Gamma(f)(b) \quad \text { for all } n>1, K \leq 1-\frac{2}{n} .
$$

Therefore, we have that this graph Laplacian satisfies the Bakry-Émery curvature-dimension condition

$$
B E\left(1-\frac{2}{n}, n\right) \text { for any } n>1,
$$

being $K=1-\frac{2}{n}$ the best constant for a fixed $n>1$.

Now, it is easy to see that gap $(-\Delta)=1$ thus, by Theorem 3.18 , we have that $\Delta$ satisfies the integrated Bakry-Émery curvature-dimension condition $\operatorname{IBE}(K, n)$ with $K=1-\frac{1}{n}>1-\frac{2}{n}$.

Note that $\Delta$ satisfies the Bakry-Émery curvature-dimension condition $B E(1, \infty)$ and hence, in this example, the bound in (3.13) is sharp but there is a gap in the bound (3.12).

Remark 3.20. For a metric random walk space $[X, d, m]$ with invariant and reversible probability measure $\nu$, Y. Ollivier in [43, Corollary 31], under the assumption that

$$
\iiint d(y, z)^{2} d m_{x}(y) d m_{x}(z) d \nu(x)<+\infty
$$

proves that if the Ollivier-Ricci curvature $\kappa_{m}$ is positive and the space is ergodic, then $[X, d, m, \nu]$ satisfies the Poincaré inequality

$$
\kappa_{m} \operatorname{Var}_{\nu}(f) \leq \mathcal{H}_{m}(f) \text { for all } f \in L^{2}(X, \nu),
$$

and, consequently,

$$
\kappa_{m} \leq \operatorname{gap}\left(-\Delta_{m}\right) .
$$

Observe that, in fact, the ergodicity follows from the positivity of $\kappa_{m}$ (Theorem 2.12). 
3.5. Transport Inequalities. Given a metric random walk space $[X, d, m]$ we define, for $x \in X$,

$$
\Theta(x):=\frac{1}{2}\left(W_{2}^{d}\left(\delta_{x}, m_{x}\right)\right)^{2}=\frac{1}{2} \int_{X} d(x, y)^{2} d m_{x}(y)
$$

and

$$
\Theta_{m}:=\underset{x \in X}{\operatorname{ess} \sup } \Theta(x) .
$$

Since $\Theta(x) \leq \frac{1}{2}\left(\operatorname{diam}\left(\operatorname{supp}\left(m_{x}\right)\right)^{2}\right.$, if $\operatorname{diam}(X)$ is finite then we have $\Theta_{m} \leq \frac{1}{2}(\operatorname{diam}(X))^{2}$. Observe that

$$
\|\Gamma(f)\|_{\infty}=\sup _{x \in X} \frac{1}{2} \int_{X}(f(x)-f(y))^{2} d m_{x}(y) \leq \Theta_{m}\|f\|_{L i p}^{2} .
$$

Given a metric measure space $(X, d, \mu)$ as in Example $1.2(4)$, if $m^{\mu, \epsilon}$ is the $\epsilon$-step random walk associated to $\mu$, that is

$$
m_{x}^{\mu, \epsilon}:=\frac{\mu \mathrm{L} B(x, \epsilon)}{\mu(B(x, \epsilon))} \quad \text { for } x \in X
$$

then

$$
\Theta_{m^{\mu, \epsilon}} \leq \frac{1}{2} \epsilon^{2}
$$

Let $J_{m}(x)$ be the jump of the random walk at $x$ :

$$
J_{m}(x):=W_{1}^{d}\left(\delta_{x}, m_{x}\right)=\int_{X} d(x, y) d m_{x}(y) .
$$

In the particular case of the metric random walk space associated to a locally finite discrete graph $\left[V(G), d_{G}, m^{G}\right]$, we have

$$
J_{m^{G}}(x)=\frac{1}{d_{x}} \sum_{y \sim x, y \neq x} w_{x y} \leq 1
$$

thus

$$
\Theta(x)=\frac{1}{2} J_{m^{G}}(x)=\frac{1}{2 d_{x}} \sum_{x \sim y, x \neq y} w_{x y} \leq \frac{1}{2} .
$$

In the case of the metric random walk space $\left[\Omega,\|\cdot\|, m^{J}\right]$ (see Example $2.3(2)$ ), with $J(x)=\frac{1}{\left|B_{r}(0)\right|} \chi_{B_{r}(0)}$, a simple computation gives

$$
\Theta(x) \leq \frac{N}{2(N+2)} r^{2} .
$$

It is well known that in the case of diffusion semigroups the Bakry-Émery curvature-dimension condition $B E(K, \infty)$ of its generator is characterized by gradient estimates on the semigroup (see for instance [5] or [6]). The same characterization is also true for weighted discrete graphs (see for instance [31] and $[26]$ ). With a similar proof we have that in the general context of metric random walk spaces this characterization is also true.

Theorem 3.21. Let $[X, d, m]$ be a metric random walk space with invariant-reversible probability measure $\nu$ and let $\left(T_{t}\right)_{t>0}=\left(e^{t \Delta_{m}}\right)_{t>0}$ be the heat semigroup. Then, $\Delta_{m}$ satisfies the Bakry-Émery curvaturedimension condition $B E(K, \infty)$ with $K>0$ if, and only if,

$$
\Gamma\left(T_{t} f\right) \leq e^{-2 K t} T_{t}(\Gamma(f)) \quad \forall t \geq 0, \forall f \in L^{2}(X, \nu) .
$$


Proof. Fix $t>0$. For $s \in[0, t)$, we define the function

$$
g(s, x):=e^{-2 K s} T_{s}\left(\Gamma\left(T_{t-s} f\right)\right)(x), \quad x \in X .
$$

The same computations as in [31] show that

$$
\frac{\partial g}{\partial s}(s, x)=2 e^{-2 K s} T_{s}\left(\Gamma_{2}\left(T_{t-s} f\right)-K \Gamma\left(T_{t-s} f\right)\right)(x) .
$$

Then, if $\Delta_{m}$ satisfies the Bakry-Émery curvature-dimension condition $B E(K, \infty)$ with $K>0$, we have that $\frac{\partial g}{\partial s}(s, x) \geq 0$ which is equivalent to (3.19). On the other hand, if (3.19) holds, we have $\frac{\partial g}{\partial s}(0, x) \geq 0$, which is equivalent to

$$
\Gamma_{2}\left(T_{t} f\right)-K \Gamma\left(T_{t} f\right) \geq 0 .
$$

Then, letting $t \rightarrow 0$, we get $\Gamma_{2}(f)-K \Gamma(f) \geq 0$. by

The Fisher-Donsker-Varadhan information of a probability measure $\mu$ on $X$ with respect to $\nu$ is defined

$$
I_{\nu}(\mu):= \begin{cases}2 \mathcal{H}_{m}(\sqrt{f}) & \text { if } \mu=f \nu, f \geq 0 \\ +\infty, & \text { otherwise. }\end{cases}
$$

Observe that

$$
D\left(I_{\nu}\right)=\left\{\mu: \mu=f \nu, f \in L^{1}(X, \nu)^{+}\right\}
$$

since $\sqrt{f} \in L^{2}(X, \nu)=D\left(\mathcal{H}_{m}\right)$ whenever $f \in L^{1}(X, \nu)^{+}$. Here, we use the notation $L^{p}(X, \nu)^{+}:=\{f \in$ $L^{p}(X, \nu): f \geq 0 \nu$-a.e. $\}$.

In the next result we show that the Bakry-Émery curvature-dimension condition $B E(K, \infty)$ with $K>0$ implies a transport-information inequality, result that was obtained for the particular case of Markov chains in discrete spaces in [24].

Theorem 3.22. Let $[X, d, m]$ be a metric random walk space with invariant-reversible probability measure $\nu$, and assume that $\Theta_{m}$ is finite. If $\Delta_{m}$ satisfies the Bakry-Émery curvature-dimension condition $B E(K, \infty)$ with $K>0$, then $\nu$ satisfies the transport-information inequality

$$
W_{1}^{d}(\mu, \nu) \leq \frac{\sqrt{\Theta_{m}}}{K} \sqrt{I_{\nu}(\mu)}, \quad \text { for all probability measures } \mu \ll \nu .
$$

Proof. Let $\mu$ be a probability measure $\mu \ll \nu$, and set $\mu=f \nu$. By the Kantorovich-Rubinstein Theorem we have that

$$
W_{1}^{d}(\mu, \nu)=\sup \left\{\int_{X} g(x)(f(x)-1) d \nu(x):\|g\|_{\text {Lip }} \leq 1 \text { and } g \text { bounded }\right\} .
$$

Let $T_{t}=e^{t \Delta_{m}}$ be the heat semigroup. Given $g \in L^{\infty}(X, \nu)$ with $\|g\|_{L i p} \leq 1$, having in mind Proposition 2.22 , we have

$$
\begin{aligned}
\int_{X} g(x)(f(x)-1) d \nu(x) & =-\int_{0}^{+\infty} \frac{d}{d t} \int_{X}\left(T_{t} g\right)(x) f(x) d \nu(x) d t=-\int_{0}^{+\infty} \int_{X} \Delta_{m}\left(T_{t} g\right)(x) f(x) d \nu(x) d t \\
& =\int_{0}^{+\infty} \mathcal{E}_{m}\left(T_{t} g, f\right) d t=\int_{0}^{+\infty} \int_{X} \Gamma\left(T_{t} g, f\right)(x) d \nu(x) d t .
\end{aligned}
$$

Now, using the Cauchy-Schwartz inequality, the reversibility of the measure $\nu$ and that

$$
(\sqrt{f}(y)+\sqrt{f}(x))^{2} \leq 2((f(x)+f(y)),
$$


we have

$$
\begin{aligned}
& \int_{X} \Gamma\left(T_{t} g, f\right)(x) d \nu(x)=\frac{1}{2} \int_{X \times X}\left(\left(T_{t} g\right)(y)-\left(T_{t} g\right)(x)\right)(f(y)-f(x)) d m_{x}(y) d \nu(x) \\
& =\frac{1}{2} \int_{X \times X}\left(\left(T_{t} g\right)(y)-\left(T_{t} g\right)(x)\right)(\sqrt{f}(y)-\sqrt{f}(x))(\sqrt{f}(y)+\sqrt{f}(x)) d m_{x}(y) d \nu(x) \\
& \leq\left(\int_{X \times X} \frac{1}{4}(\sqrt{f}(y)-\sqrt{f}(x))^{2} d m_{x}(y) d \nu(x)\right)^{\frac{1}{2}} \\
& \quad \times\left(\int_{X \times X}\left(\left(T_{t} g\right)(y)-\left(T_{t} g\right)(x)\right)^{2}(\sqrt{f}(y)+\sqrt{f}(x))^{2} d m_{x}(y) d \nu(x)\right)^{\frac{1}{2}} \\
& \leq\left(\frac{1}{2} \int_{X} \Gamma(\sqrt{f})(x) d \nu(x)\right)^{\frac{1}{2}}\left(4 \int_{X}\left(\int_{X}\left(\left(T_{t} g\right)(y)-\left(T_{t} g\right)(x)\right)^{2} d m_{x}(y)\right) f(x) d \nu(x)\right)^{\frac{1}{2}} .
\end{aligned}
$$

Then, applying Theorem 3.21, we get

$$
\begin{gathered}
\int_{X} g(x)(f(x)-1) d \nu(x) \leq\left(\frac{1}{2} \mathcal{H}_{m}(\sqrt{f})\right)^{\frac{1}{2}} \int_{0}^{+\infty}\left(4 \int_{X} \Gamma\left(\left(T_{t} g\right)(x)\right) f(x) d \nu(x)\right)^{\frac{1}{2}} d t \\
\leq\left(2 \mathcal{H}_{m}(\sqrt{f})\right)^{\frac{1}{2}} \int_{0}^{+\infty}\left(e^{-2 K t} \int_{X} T_{t}(\Gamma(g))(x) f(x) d \nu(x)\right)^{\frac{1}{2}} d t
\end{gathered}
$$

Now, by (3.18) and (2.5), we have

$$
\left|T_{t}(\Gamma(g))(x)\right| \leq\left\|T_{t}(\Gamma(g))\right\|_{\infty} \leq\|\Gamma(g)\|_{\infty} \leq \Theta_{m} .
$$

Hence

$$
\int_{X} g(x)(f(x)-1) d \nu(x) \leq\left(2 \mathcal{H}_{m}(\sqrt{f})\right)^{\frac{1}{2}} \int_{0}^{+\infty}\left(e^{-2 K t} \Theta_{m} \int_{X} f(x) d \nu(x)\right)^{\frac{1}{2}} d t \leq \frac{\sqrt{\Theta_{m}}}{K}\left(2 \mathcal{H}_{m}(\sqrt{f})\right)^{\frac{1}{2}}
$$

Finally, taking the supremum over all bounden functions $g$ with $\|g\|_{\text {Lip }} \leq 1$ we get (3.20).

Remark 3.23. If $\nu$ satisfies a transport-information inequality

$$
W_{1}^{d}(\mu, \nu) \leq \lambda \sqrt{2 \mathcal{H}_{m}(\sqrt{f})} \quad \text { for all } \mu=f \nu,
$$

then $\nu$ is ergodic. In fact, if $\nu$ is not ergodic, then by Theorem 2.24 there exists $D \subset X$ with $0<\nu(D)<1$ such that $\Delta_{m} \chi_{D}=0$. Now, if $\mu:=\frac{1}{\nu(D)} \chi_{D} \nu$, then $\mu \neq \nu$ and, therefore, by (3.21), we get $\mathcal{H}_{m}\left(\chi_{D}\right)>0$, which is a contradiction with $\Delta_{m} \chi_{D}=0$.

As a consequence of the previous Remark and Theorem 3.22, we have that the positivity of the BakryÉmery curvature-dimension condition implies ergodicity of $\Delta_{m}$, then, by Theorem 3.18, we have the following result.

Theorem 3.24. Let $[X, d, m]$ be a metric random walk space with invariant-reversible probability measure $\nu$, and assume that $\Theta_{m}$ is finite. Then: 
If $\Delta_{m}$ satisfies the Bakry-Émery curvature-dimension condition $B E(K, n)$ with $K>0$, we have

$$
\operatorname{gap}\left(-\Delta_{m}\right) \geq K \frac{n}{n-1} .
$$

In the case that $\Delta_{m}$ satisfies the Bakry-Émery curvature-dimension condition $B E(K, \infty)$ with $K>0$, we have

$$
\operatorname{gap}\left(-\Delta_{m}\right) \geq K
$$

The relative entropy of $0 \leq \mu \in \mathcal{M}(X)$ with respect to $\nu$ is defined by

$$
\operatorname{Ent}_{\nu}(\mu):= \begin{cases}\int_{X} f \log f d \nu-\nu(f) \log (\nu(f)) & \text { if } \mu=f \nu, f \geq 0, f \log f \in L^{1}(X, \nu), \\ +\infty, & \text { otherwise, }\end{cases}
$$

with the usual convention that $f(x) \log f(x)=0$ if $f(x)=0$.

The next result shows that a transport-information inequality implies a transport-entropy inequality and, therefore, normal concentration (see for example [13, 32]).

Theorem 3.25. Let $[X, d, m]$ be a metric random walk space with invariant-reversible probability measure $\nu$ and assume that $\Theta_{m}$ is finite and that there exists some $x_{0} \in X$ such that $\int d\left(x, x_{0}\right) d \nu(x)<\infty$. Then the transport-information inequality

$$
W_{1}^{d}(\mu, \nu) \leq \frac{1}{K} \sqrt{I_{\nu}(\mu)}, \quad \text { for all probability measures } \mu \ll \nu,
$$

implies the transport-entropy inequality

$$
W_{1}^{d}(\mu, \nu) \leq \sqrt{\frac{\sqrt{2 \Theta_{m}}}{K} \operatorname{Ent}_{\nu}(\mu)}, \quad \text { for all probability measures } \mu \ll \nu .
$$

Proof. By [13, Theorem 1.3], inequality (3.23) is equivalent to

$$
\int_{X} e^{\lambda f(x)} d \nu(x) \leq e^{\lambda^{2} \frac{\sqrt{\Theta m}}{2 \sqrt{2} K}}
$$

for every bounded function $f$ on $X$ with $\|f\|_{L i p} \leq 1$ and $\nu(f)=0$, and all $\lambda \in \mathbb{R}$.

Given $f \in L^{\infty}(X, \nu)$ with $\|f\|_{L i p} \leq 1$ and $\nu(f)=0$, we define the function

$$
\Lambda(\lambda):=\int_{X} e^{\lambda f(x)} d \nu(x)
$$

and the probabilities

$$
\mu_{\lambda}:=\frac{1}{\Lambda(\lambda)} e^{\lambda f} d \nu
$$

By the Kantorovich-Rubinstein Theorem and the assumption (3.22), we have

$$
\begin{gathered}
\frac{d}{d \lambda} \log (\Lambda(\lambda))=\frac{1}{\Lambda(\lambda)} \int_{X} f(x) e^{\lambda f(x)} d \nu(x)=\int_{X} f(x)\left(d \mu_{\lambda}(x)-d \nu(x)\right) \leq W_{1}^{d}\left(\mu_{\lambda}, \nu\right) \leq \\
\leq \frac{1}{K} \sqrt{2 \mathcal{H}_{m}\left(\sqrt{\frac{1}{\Lambda(\lambda)} e^{\lambda f}}\right)}=\frac{\sqrt{2}}{K} \sqrt{\int_{X} \Gamma\left(\sqrt{\frac{1}{\Lambda(\lambda)} e^{\lambda f}}\right)(x) d \nu(x)}
\end{gathered}
$$




$$
=\frac{\sqrt{2}}{K} \sqrt{\int_{X} \frac{1}{\Lambda(\lambda)} \Gamma\left(e^{\frac{\lambda f}{2}}\right)(x) d \nu(x)} .
$$

Now, since $1-\frac{1}{a} \leq \log a$ for $a \geq 1$, having in mind the reversibility of $\nu$, we have

$$
\int_{X} \Gamma(g)(x) d \nu(x) \leq \int_{X} g^{2}(x) \Gamma(\log g)(x) d \nu(x),
$$

and, consequently, by (3.18), we get

$$
\begin{gathered}
\frac{d}{d t} \log (\Lambda(\lambda)) \leq \frac{\sqrt{2}}{K} \sqrt{\int_{X} \frac{1}{\Lambda(\lambda)} e^{\lambda f(x)} \Gamma\left(\frac{\lambda f}{2}\right)(x) d \nu(x)}=\frac{\lambda}{\sqrt{2} K} \sqrt{\int_{X} \frac{1}{\Lambda(\lambda)} e^{\lambda f(x)} \Gamma(f)(x) d \nu(x)} \\
=\frac{\lambda}{\sqrt{2} K} \sqrt{\int_{X} \Gamma(f)(x) d \mu_{\lambda}(x)} \leq \frac{\sqrt{\Theta_{m}}}{\sqrt{2} K} \lambda .
\end{gathered}
$$

Then, integrating we get (3.24).

In the next example we see that, in general, a transport-entropy inequality does not imply transportinformation inequality.

Example 3.26. Let $\Omega=[-1,0] \cup[2,3]$ and consider the metric random walk space $\left[\Omega, d, m^{J, \Omega}\right]$, with $d$ the Euclidean distance in $\mathbb{R}$ and $J(x)=\frac{1}{2} \chi_{[-1,1]}$ (see Example (1.2) (5)). An invariant and reversible probability measure for $m^{J, \Omega}$ is $\nu:=\frac{1}{2} \mathcal{L}^{1}\llcorner\Omega$. By the Gaussian integrability criterion [20, Theorem 2.3] $\nu$ satisfies a transport-entropy inequality. However, $\nu$ does not satisfy a transport-information inequality, since if $\nu$ satisfies a transport-information inequality, then $\nu$ must be ergodic (see Remark 3.23). Now it is easy to see that $\left[\Omega, d, m^{J, \Omega}\right]$ is not $m$-connected and then by Theorem $2.19, \nu$ is not ergodic.

By Theorems 2.12 and 2.19 , we have that the metric random walk space $\left[\Omega, d, m^{J, \Omega}\right]$ of the above example has non-positive Ollivier-Ricci curvature. In the next theorem we will see that, under positive Ollivier-Ricci curvature, a transport-information inequality holds. First we need the following result.

Lemma 3.27. Let $[X, d, m]$ be a metric random walk space with invariant-reversible probability measure $\nu$. Then, if $f \in L^{2}(X, \nu)$ with $\|f\|_{L i p} \leq 1$, we have $\left\|e^{t \Delta_{m}} f\right\|_{L i p} \leq e^{-t \kappa_{m}}$.

Proof. By [43, Proposition 25], we have that

$$
\kappa_{m^{*(n+l)}} \geq \kappa_{m^{* n}}+\kappa_{m^{* l}}-\kappa_{m^{* n}} \kappa_{m^{* l}} \quad \forall n, l \in \mathbb{N} .
$$

where $\kappa_{m^{* 1}}=\kappa_{m}$. Hence,

$$
1-\kappa_{m^{* n}} \leq\left(1-\kappa_{m}\right)^{n} \quad \forall n \in \mathbb{N} .
$$

By Theorem 2.4 and (3.25), we have

$$
\begin{gathered}
\left|e^{t \Delta_{m}} f(x)-e^{t \Delta_{m}} f(y)\right|=\left|e^{-t} \sum_{n=0}^{+\infty} \int_{X} f(z)\left(d m_{x}^{* n}(z)-d m_{y}^{* n}(z)\right) \frac{t^{n}}{n !}\right| \\
\leq e^{-t} \sum_{n=0}^{+\infty} W_{1}^{d}\left(m_{x}^{* n}, m_{y}^{* n}\right) \frac{t^{n}}{n !} \leq e^{-t} \sum_{n=0}^{+\infty}\left(1-\kappa_{m^{* n}}\right) d(x, y) \frac{t^{n}}{n !} \leq e^{-t} \sum_{n=0}^{+\infty}\left(1-\kappa_{m}\right)^{n} \frac{t^{n}}{n !} d(x, y) \\
=e^{-t} e^{t\left(1-\kappa_{m}\right)} d(x, y)=e^{-t \kappa_{m}} d(x, y),
\end{gathered}
$$

from where it follows that $\left\|e^{t \Delta_{m}} f\right\|_{L i p} \leq e^{-t \kappa_{m}}$. 
Theorem 3.28. Let $[X, d, m]$ be a metric random walk space with invariant-reversible probability measure $\nu$, and assume that $\Theta_{m}$ is finite. If $\kappa_{m}>0$ then the following transport-information inequality holds:

$$
W_{1}^{d}(\mu, \nu) \leq \frac{\sqrt{2 \Theta_{m}}}{\kappa_{m}} \sqrt{I_{\nu}(\mu)}, \quad \text { for all probability measures } \mu \ll \nu \text {. }
$$

Proof. Let $T_{t}=e^{t \Delta_{m}}$ be the heat semigroup and $\mu=f \nu$ be a probability measure in $X$. We use, as in the proof of Theorem 3.22, the Kantorovich-Rubinstein Theorem. Let $g \in L^{\infty}(X, \nu)$ with $\|g\|_{L i p} \leq 1$. Having in mind Lemma 3.27, we have

$$
\begin{gathered}
\int_{X} g(x)(f(x)-1) d \nu(x)=-\int_{0}^{+\infty} \frac{d}{d t} \int_{X}\left(T_{t} g\right)(x) f(x) d \nu(x) d t=-\int_{0}^{+\infty} \int_{X} \Delta_{m}\left(T_{t} g\right)(x) f(x) d \nu(x) d t \\
=\int_{0}^{+\infty} \frac{1}{2} \int_{X \times X}\left(\left(T_{t} g\right)(y)-\left(T_{t} g\right)(x)\right)(f(y)-f(x)) d m_{x}(y) d \nu(x) d t \\
\leq \int_{0}^{+\infty}\left\|T_{t} g\right\|_{L i p} \frac{1}{2} \int_{X \times X} d(x, y)|f(y)-f(x)| d m_{x}(y) d \nu(x) d t \\
\leq \int_{0}^{+\infty} e^{-t \kappa_{m}} \frac{1}{2} \int_{X \times X} d(x, y)|f(y)-f(x)| d m_{x}(y) d \nu(x) d t \\
=\frac{1}{2 \kappa_{m}} \int_{X \times X} d(x, y)|\sqrt{f}(y)-\sqrt{f}(x)|(\sqrt{f}(y)+\sqrt{f}(x)) d m_{x}(y) d \nu(x) \\
\leq \frac{\sqrt{2}}{2 \kappa_{m}} \sqrt{\mathcal{H}_{m}(\sqrt{f})} \sqrt{\int_{X \times X} d^{2}(x, y)(\sqrt{f}(y)+\sqrt{f}(x))^{2} d m_{x}(y) d \nu(x)} .
\end{gathered}
$$

Now, using reversibility of $\nu$,

$$
\begin{gathered}
\int_{X \times X} d^{2}(x, y)(\sqrt{f}(y)+\sqrt{f}(x))^{2} d m_{x}(y) d \nu(x) \\
=\int_{X \times X} d^{2}(x, y)\left(2 f(x)+2 f(y)-(\sqrt{f}(y)-\sqrt{f}(x))^{2}\right) d m_{x}(y) d \nu(x) \\
\leq 2 \int_{X \times X} d^{2}(x, y)(f(x)+f(y)) d m_{x}(y) d \nu(x) \leq 8 \Theta_{m} .
\end{gathered}
$$

Therefore, we get

$$
\int_{X} g(x)(f(x)-1) d \nu(x) \leq \frac{2 \sqrt{\Theta_{m}}}{\kappa_{m}} \sqrt{\mathcal{H}_{m}(\sqrt{f})},
$$

so, taking the supremum over the functions $g$,

$$
W_{1}^{d}(\mu, \nu) \leq \frac{\sqrt{2 \Theta_{m}}}{\kappa_{m}} \sqrt{2 \mathcal{H}_{m}(\sqrt{f})}=\frac{\sqrt{2 \Theta_{m}}}{\kappa_{m}} \sqrt{I_{\nu}(\mu)} .
$$

Acknowledgment. The authors have been partially supported by the Spanish MCIU and FEDER, project PGC2018-094775-B-I00. The second author was also supported by Ministerio de Economía y Competitividad under Grant BES-2016-079019. 


\section{REFERENCES}

[1] L. Ambrosio, N. Fusco and D. Pallara, Functions of Bounded Variation and Free Discontinuity Problems. Oxford Mathematical Monographs, 2000.

[2] N. Alon and V.D. Milman, $\lambda_{1}$, Isoperimetric inequalities for graphs, and superconcentrators. J. Combin. Theory Ser. B 38, (1985) 73-88.

[3] L. Ambrosio, N. Gigli and G. Savaré, Bakry-Émery curvature-dimension condition and Riemannian Ricci curvature bounds. Ann. Probab. 43 (2015), 339-404.

[4] F. Andreu-Vaillo, J. M. Mazón, J. D. Rossi and J. Toledo, Nonlocal Diffusion Problems. Mathematical Surveys and Monographs, vol. 165. AMS, 2010.

[5] D. Bakry, Functional inequalities for Markov semigroups. In: Probability Measures on Groups: Recent Directions and Trends, pp. 91-147. Tata Institute of Fundamental Research, Mumbai (2006)

[6] D. Bakry, I. Gentil and M. Ledoux, Analysis and Geometry of Markov Diffusion Operators. Grundlehren der Mathematischen Wissenschafter, 348, Springer, 2014.

[7] D. Bakry and M. Émery, Diffusions hypercontractives. (French) [Hypercontractive diffusions] Séminaire de probabilités, XIX, 1983/84, 177-206, Lecture Notes in Math., 1123, Springer, Berlin, 1985.

[8] B. B. Bhattacharya and S. Mukherjee, Exact and asymptotic results on coarse Ricci curvature of graphs. Discrete Mathematics 338 (2015), 23-42.

[9] F. Bauer, F. Chung, Y. Lin and Y. Liu, Curvature aspect of Graphs. Proc. Amer. Math. Soc. 145 (2017), 2033-2042.

[10] F. Bauer and J. Jost, Bipartite and neighborhood graps and the spectrum of the normalized graph laplace operator. Comm. in Analysis and geometry 21 (2013), 787-845.

[11] F. Bauer, J. Jost and S. Liu, Ollivier-Ricci Curvature and the spectrum of the normalized graph Laplace operator. Math. Res. Lett. 19 (2012), 1185-1205.

[12] Ph. Bénilan and M. G. Crandall, Completely Accretive Operators. In Semigroups Theory and Evolution Equations (Delft, 1989), Ph. Clement et al. editors, volume 135 of Lecture Notes in Pure and Appl. Math., Marcel Dekker, New York, 1991, pp. 41-75.

[13] S.G. Bobkov and F. Götze, Exponential Integrability and Transportation Cost Related to Logarithmic Inequalities. Journal of Functional Analysis 163 (1999), 1-28.

[14] S.G. Bobkov and P. Tetali, Modified Logarithmic Sobolev Inequalities in Discrete Setting. Journal of Theoretical Probability 19 (2006), 289-336.

[15] H. Brezis, Operateurs Maximaux Monotones. North Holland, Amsterdam, 1973.

[16] H. Brezis, Functional Analysis, Sobolev Spaces and Partial Differential equations. Universitext, Springer, 2011.

[17] J. Cheeger, A lower bound for the smallest eigenvalue of the Laplacian, Problems in Analysis, (R. C. Gunning, ed.) Princeton Univ. Press (1970) 195-199.

[18] H. J. Cho and S-H. Paeng, Olivier's Ricci curvature and coloring. European Journal of Combinatorics 34 (2013), 916-922.

[19] F. Chung, Spectral Graph Theory (CBMS Regional Conference Series in Mathematics, No. 92), American Mathematical Society, 1997.

[20] H. Djellout, A. Guillin, and L.Wu, Transportation cost-information inequalities for random dynamical systems and diffusions. Annals of Probability, 32 (2004), 2702-2732.

[21] J. Dodziuk, Difference equations, isoperimetric inequality and transience of certain random walks. Trans. Amer. Math. Soc. 284 (1984), 787-794.

[22] J. Dodziuk and L. Karp, Spectral and Function Theory for Combinatorial Laplacian. Comptemp. Math. vol. 73. American Mathematical Society, Providence, RI, 2001.

[23] M. Erbas and J. Maas, Ricci Curvature of Finite Markov Chains via Convexity of the Entropy. Arch. Rat. Mech. Anal. 206 (2012), 997-1038.

[24] M. Fathi and Y. Shu, Curvature and transport inequalities for Markov chains in discrete spaces. Bernouilli 24 (2018), $672-698$.

[25] M. Fukushima, Y. Oshima and M. Takeda. Dirichlet forms and symetric Markov processes, Studies in Mathematics 19, De Gruyter, 2011.

[26] Ch. Gong and Y. Lin, Equivalent properties for CD inequalities on graphs with unbounded Laplacians. Chin. Ann. Math. Ser. B 38 (2017), 1059-1070.

[27] N. Gozlan and C. Léonard, Transport inequalities. A survey. Markov Process. Related Fields 16 (2010), 635-736.

[28] N. Gozlan, C. Roberto, P-M. Samson and P. Tetali, Displacement convexity of entropy and related inequalities on graphs. Probab. Theory and Related Fields 160 (2014), 47-94.

[29] O. Hernández-Lerma and J. B. Laserre, Markov Chains and Invariant Probabilities. Birkhäuser Verlag, Basel, 2003. 
[30] J. Jost and S. Liu, Ollivier's Ricci Curvature, Local Clustering and Curvature-Dimension Inequalities on Graphs. Discrete Compt. Geom. 51 (2014), 300-322.

[31] B. Klartag, G. Kozma, P. Ralli and P. Tetali, Discrete Curvature and Abelian Groups. Canad. J. Math. 68 (2016), 655-674.

[32] M. Ledoux, The Concentration of Measures Phenomenon. Math Surveys and Monographs. Vol 89, Amer. Math.Soc, 2001.

[33] Y. Lin and and S-T. Yau, Ricci Curvature and eigenvalue estimates on locally finite graphs. Math. Res. Lett. 17 (2010), 343-356.

[34] J. Lott and C. Villani, Ricci curvature for metric-measure spaces via optimal transport. Ann. of Math. 169 (2009), 903-991.

[35] K. Marton, A simple proof of the blowing-up lemma. IEEE Trans. Inform. Theory, 32 (1986), 445-446.

[36] K. Marton, A measure concentration inequality for contracting Markov chains. Geom. Funct. Anal., 6 (1996), 556-571.

[37] J. Maas, Gradient flows of the entropy for finite Markov chains. J.Funct.Anal. 261 (2011), 2250-2292.

[38] J. M. Mazón, J. D. Rossi and J. Toledo, The Heat Content for Nonlocal Diffusion with Non-singular Kernels. Adv. Nonlinear Stud. 17 (2017), 255-268.

[39] J. M. Mazón, M. Solera and J. Toledo, The Total Variation Flow in Metric Random Walk Spaces. arXiv:1905.01130

[40] J. M. Mazón, M. Solera and J. Toledo, Gradient Flows and Ricci Curvature on Metric Random Walk Spaces. Forthcoming paper.

[41] L. Miclo, On hyperboundedness and spectrum of Markov operators. Invent. math. 200 (2015), 311-343.

[42] L. Najman and P. Romon (ed.), Modern Approaches to Discrete Curvature. Lecture Notes in Mathematics 2184, Springer, 2017.

[43] Y. Ollivier, Ricci curvature of Markov chains on metric spaces. J. Funct. Anal. 256 (2009), 810-864.

[44] Y. Ollivier, A survey of Ricci curvature for metric spaces and Markov chains. Probabilistic approach to geometry, 343-381, Adv. Stud. Pure Math., 57, Math. Soc. Japan, Tokyo, 2010.

[45] Y. Ollivier and C. Villani, A curved Brunn-Minkowski inequality on the discrete hypercube, or: what is the Ricci curvature of the discrete hypercube? SIAM J. Discrete Math. 26 (2012), 983-996.

[46] S-H. Paeng, Volume and diametrer of a grap anf Olivier's Ricci curvature. European Journal of Combinatorics 33 (2012), 1808-1819.

[47] G. Polya and S. Szego, Isoperimetric Inequalities in Mathematical Physics, Annals of Math. Studies, no. 27, Princeton University Press, (1951).

[48] M. Reed and B. Simon. Functional Analalysis I. Academic Press 1980.

[49] M.-K von Renesse. K-L. Sturm, Transport Inequalities, Gradient Estimates, Entropy, and Ricci Curvature, Communications on Pure and Applied Mathematics, Vol. LVIII, (2005), 0923-0940.

[50] M. Schmuckenschlager, Curvature of Nonlocal Markov Generators. In Convex Geometric Analysis (Berkeley, CA, 1996), Cambrigge Univ. Press. 1998, pp. 189-197.

[51] K-L. Sturm, On the geometry of metric measure spaces. I, II. Acta Math. 196 (2006), 65-131 and 133-177.

[52] A. Szlam and X. Bresson, Total Variation and Cheeger Cuts. Proceedings of the 27 th International Confer- ence on Machine Learning, Haifa, Israel, 2010.

[53] M. Talagrand, Transportation cost for Gaussian and other product measures. Geom. Funct. Anal., 6 (1996), 587-600.

[54] C. Villani. Topics in Optimal Transportation. Graduate Studies in Mathematics. Vol. 58, 2003.

[55] C. Villani. Optimal transport. old and new, Grundlehren der Mathematischen Wissenschaften, vol. 338, Springer-Verlag, Berlin, 2009.

J. M. Mazón: Departamento de Análisis Matemático, Univ. Valencia, Dr. Moliner 50, 46100 Burjassot, SPAIN. mazon@uv.es

M. Solera: Departamento de Análisis Matemático, Univ. Valencia, Dr. Moliner 50, 46100 Burjassot, Spain. marcos.solera@uv.es

J. Toledo: Departamento de Análisis Matemático, Univ. Valencia, Dr. Moliner 50, 46100 Burjassot, Spain. toledojj@uv.es 